\title{
REGENERAÇÃO NATURAL PÓS-FOGO NOS CAMPOS DE ALTITUDE NO PARQUE NACIONAL DO ITATIAIA, SUDESTE DO BRASIL
}

\author{
Izar Aximoff ${ }^{1 *}$, André Felippe Nunes-Freitas ${ }^{2}$ \& João Marcelo Alvarenga Braga ${ }^{1}$ \\ ${ }^{1}$ Instituto de Pesquisas Jardim Botânico do Rio de Janeiro. Rua Pacheco Leão, 915. CEP 22460-030. Rio de Janeiro, RJ, Brasil. \\ ${ }^{2}$ Universidade Federal Rural do Rio de Janeiro, Instituto de Florestas, Departamento de Ciências Ambientais. Laboratório de \\ Ecologia Florestal e Biologia Vegetal, DCA/IF, Rodovia BR-465, Km 7 Br 465. Campus Universitário. Seropédica, RJ, Brasil. \\ CEP: 23890-000. \\ E-mails: izar.aximoff@gmail.com,afnfreitas@gmail.com,jmabraga@jbrj.gov.br
}

\section{RESUMO}

O fogo descontrolado (i.e. incêndio) de origem antrópica é a ameaça mais frequente nos campos de altitude no Estado do Rio de Janeiro. Mais de 70\% das ocorrências nesse ecossistema foram registradas no interior do Parque Nacional do Itatiaia - PNI. No entanto seus impactos ainda são pouco conhecidos. Compreender os processos associados à regeneração da vegetação de campos de altitude pós-fogo é sugerido pelo Plano de Manejo do PNI. Assim, o objetivo deste estudo foi avaliar a composição florística e a estrutura da comunidade vegetal em quatro sítios localizados a 2500m de altitude e com diferentes históricos pós-fogo em cada um (1988, 2001, 2007 e 2010). Foi estudada uma área de $1600 \mathrm{~m}^{2}$ dividida entre fisionomias campestre graminóide e ilhas de vegetação sobre afloramentos rochosos. No total, foram amostradas 167 espécies de angiospermas e 16 de samambaias e licófitas, sendo 32\% endêmicas do Sudeste do Brasil e 11\% ameaçadas de extinção. Asteraceae apresentou quase $20 \%$ das espécies dentre as 63 famílias registradas. Entre as fisionomias, houve similaridade florística intra e entre sítios $(>0,70)$. Comparando as duas fisionomias, os valores de similaridade foram em geral mais baixos $(<0,70)$. A forma de vida hemicriptófita e o hábito herbáceo foram dominântes (área de cobertura) nas duas fisionomias. A riqueza entre quatro e sete anos pós-fogo teve aumento significativo (30\% das espécies), com sítio 2010 apresentando 57,4\% das espécies registradas e os demais sítios com valores acima de $80 \%$. A regeneração da vegetação ocorreu relativamente rápido, fazendo com que sítios entre quatro e sete anos de regeneração tenham apresentado resultados similares da estrutura, composição e riqueza, do sítio com 25 anos de regeneração. Contudo, algumas das espécies endêmicas e ameaçadas de extinção não foram registradas pós-fogo em alguns sítios ou apresentaram frequência entre as mais baixas. Considerando que algumas destas espécies podem estar em processo de desaparecimento, estudos populacionais, reprodutivos e ecofisiológicos tornam-se prioritários, assim como estudos sobre ecologia do fogo neste tipo de ambiente.

Palavras chave: distúrbio antrópico; espécies ameaçadas; incêndios; Serra da Mantiqueira.

\section{ABSTRACT - POST-FIRE NATURAL REGENERATION OF HIGH ALTITUDE GRASSLANDS IN THE ITATIAIA NATIONAL PARK, SOUTHEAST OF BRAZIL}

The anthropogenic fire is a major threat to the high altitude grasslands of the Itatiaia National Park - INP, where over $70 \%$ of cases of fire in this ecosystem in the state of Rio de Janeiro. However, their impacts are still unknown. Understand the processes associated with the regeneration of high altitude grasslands after fire is suggested by the Management Plan of INP. The objective of this study was to evaluate the floristic composition and structure of the plant community in four sites located at $2500 \mathrm{~m}$ altitude and with different historical fires $(1988,2001,2007$ and 2010). It was studied area of $1600 \mathrm{~m}^{2}$ divided between grassland and vegetation islands on rocky outcrops. In total were sampled 167 species of angiosperms and 16 ferns and lycophytes, with $32 \%$ endemic species and $11 \%$ threatened. Asteraceae showed nearly $20 \%$ of the species among the 63 registered families. There was floristic similarity within and between sites comparing the two vegetation types, with rocky outcrops showing lower values. Life forms, habit and dominance (coverage area) were also similar between sites. The number of species from four to seven years after fire had a significant increase (30\% of species), with sites in 1988, 2001 and 2007 with values above $80 \%$ of the recorded species. Despite of the re-establishment, structure, composition and richness of vegetation have occurred relatively quickly (between 4 and 7 years), endemic and endangered species were not found after fire. Whereas some of these threatened species can be in the disappearance process, the execution of population studies, reproductive and ecophysiological becomes a priority, as well as studies on the fire ecology in this environment.

Keywords: anthropogenic disturbance; endangered species; fire; Serra da Mantiqueira. 


\section{INTRODUÇÃO}

Um dos ecossistemas associados ao Bioma Mata Atlântica, os campos de altitude ocorrem de maneira insular no topo das montanhas mais altas do Sudeste e Sul do Brasil, com distribuição ao longo das Serras da Mantiqueira (incluindo a Serra do Caparaó) e do Mar, entre 1600-1800m de altitude (Martinelli 1996, Safford 1999a, Caiafa \& Silva 2007, Mocochinski \& Scheer 2008). A vegetação é constituída por matriz campestre, onde predominam gramíneas, pequenos arbustos, algumas arvorestas e ervas, e também por espécies que ocorrem em ilhas de vegetação sobre afloramentos rochosos (Safford 1999a, Medina et al. 2006, Ribeiro et al. 2007). Esse ecossistema vem sendo estudado em relação à composição florística (Ribeiro et al. 2007, Mocochinski \& Scheer 2008, Aximoff \& Ribeiro 2012, Meireles et al. 2014), fisionomias e condições climáticas (Safford 1999a, b), ao efeito do fogo (Safford 2001), à fenologia e relação planta-animal (Freitas \& Sazima 2006), relação planta-planta e plantatopografia (Medina et al. 2006), e à ecofisiologia de espécies arbustivas (Scarano et al. 2001). O elevado número de espécies endêmicas, raras e ameaçadas (Martinelli 1996, Aximoff 2011) e o papel deste ecossistema na regulação do ciclo hidrológico, confere importância social e ambiental, colocando-o como prioritário para a conservação (Beniston 2006, Kohler et al. 2010).

Embora ocorram em outros Estados das regiões sudeste e sul do Brasil (Robim et al. 1990, Freitas \& Sazima 2006, Caiafa \& Silva 2007, Mocochinski \& Scheer 2008, Meireles et al. 2014), no Estado do Rio de Janeiro a maior parte dos campos de altitude encontram-se no interior de unidades de conservação (UC) e, mesmo assim, enfrentam impactos antrópicos, com destaque para aqueles causados pelo fogo (Aximoff 2011). Como qualquer fator de distúrbio em um ambiente natural, os efeitos do fogo sobre a biodiversidade dependem da frequência, amplitude e época de ocorrência (Aximoff 2011). Em geral, o fogo pode levar a um processo de substituição de espécies, com maior predominância daquelas mais adaptadas (Hooper et al. 2004, Vieira \& Scariot 2006), embora mesmo estas espécies com atributos que favorecem seu estabelecimento pós-fogo (Fidelis et al. 2008), podem não sobreviver a ocorrência de fogo freqüente.

Apesar das ocorrências de paleoincêndios nos campos de altitude, desde pelo menos $20 \mathrm{mil}$ anos atrás, antes mesmo da chegada dos seres humanos (Behling 1997, Behling et al. 2007, Behling and Safford 2010, Veríssimo et al. 2012), há mais de um século o fogo é relatado com maior frequência e com raras exceções relacionado a eventos naturais (Aximoff 2011, Aximoff \& Rodrigues 2011), diferentemente do que ocorre no cerrado e nos campos rupestres (Moreira 2000, Conceição \& Pirani 2005, Kolbek \& Alves 2008, Miranda et al. 2009, Neves \& Conceição 2010). Por outro lado, algumas características dos campos de altitude como o clima seco, geadas, ventos constantes e grande quantidade de biomassa vegetal dessecada durante o inverno (Brade 1956, Safford 1999a) facilitam a ocorrência e propagação do fogo.

Nas Serras dos Órgãos - RJ e do Caparaó ES, mediante ocorrência de fogo antrópico, Safford (2001) registrou rápida regeneração natural dos campos de altitude considerando a heterogeneidade ambiental. Aximoff (2011) em estudo que levantou as ocorrências do fogo, sua periodicidade e as espécies ameaçadas em todos os campos de altitude do Estado do Rio de Janeiro, identificou que o Parque Nacional do Itatiaia (PNI) concentrou cerca de $70 \%$ destas ocorrências. No entanto, estudos da regeneração natural da vegetação pósfogo ainda não foram realizados, sendo, portanto, considerados prioritários pelo Plano de Manejo do PNI (ver Encarte 4 pags. 48-50, ICMBio 2013).

Desta forma, o objetivo deste estudo foi conhecer a composição e a estrutura da vegetação campestre e rupícola de quatro sítios atingidos por fogo antrópico em diferentes anos (1988, 2001, 2007 e 2010) nos campos de altitude no Parque Nacional do Itatiaia.

\section{MATERIAL E MÉTODOS}

Área de estudo

O Parque Nacional do Itatiaia (PNI) está localizado no sul dos Estados do Rio de Janeiro e Minas Gerais $\left(22^{\circ} 15^{\prime}\right.$ e $22^{\circ} 30^{\prime} \mathrm{S}, 4^{\circ} 30^{\prime}$ e $\left.44^{\circ} 45^{\prime} \mathrm{W}\right)$, na parte mais elevada da Serra da 
Mantiqueira (Figura 1). A importância biológica desta unidade de conservação de proteção integral, inserida no Domínio da Floresta Atlântica (Oliveira-Filho \& Fontes 2000), está relacionada ao extenso gradiente altitudinal de mais de 2000 metros de altitude (mínimo de 600 e máximo de $2792 \mathrm{~m}$ ), que possibilita a ocorrência de diferentes fitosionomias relacionadas a Floresta Ombrófila Densa, como florestas montana e alto montana, além dos campos de altitude, que são encontrados acima do limite florestal, a partir de 1800-2000m de altitude (IBGE 2012).

Os campos de altitude do PNI apresentam altitude média de $2500 \mathrm{~m}$, onde predomina matriz campestre graminóide em extensão de cerca de 10 mil ha no planalto do Itatiaia, sendo a maior área contínua existente deste ecossistema (Aximoff 2011). Espécies herbáceo-arbustiva, geralmente com até um metro de altura, ocorrem entremeadas nos blocos ou em lajes de pedra, estando dispostas em ilhas de vegetação de diferentes formas e tamanhos. Ainda, é possível indentificar na matriz campestre alguns trechos formados por arbustos e arvoretas que podem chegar a quatro metros de altura, sob as quais ocorre uma vegetação herbácea densa. A composição florística específica deste ambiente se deve ao isolamento no alto das montanhas e a fatores ambientais limitantes, como temperaturas baixas, geadas constantes, seca durante o inverno, solo pedregoso e raso, que representam uma barreira para que espécies florestais se estabeleçam (Safford 1999a, b). O clima é Cwb-mesotérmico (Köppen 1948), para as áreas acima de $1600 \mathrm{~m}$ de altitude, com verão brando que constitui a estação chuvosa, para onde a precipitação anual registrada é de $2600 \mathrm{~mm}$ (ICMBio 2013).

\section{Amostragem}

O estudo foi desenvolvido entre julho de 2013 e junho de 2014, em campo de altitude localizado no planalto do Itatiaia a $2500 \mathrm{~m}$ de altitude. Nos últimos 14 anos foram registradas três grandes queimadas $(2001=741,6 \mathrm{ha} ; 2007=763,8$ ha; $2010=1023,3$ ha) sobrepostos a $81 \%$ da área queimada em 1988. Anteriormente, em 1963, houve o maior e mais duradouro incêndio que atingiu toda área de campos de altitude e parte da área florestal.
Informações das outras 89 ocorrências no PNI podem ser obtidas em Aximoff \& Rodriguez (2011) e Aximoff (2011). Os sítios atingidos em cada uma destas três últimas grandes ocorrências de fogo e ainda um pequeno sítio queimado em 1988 foram estudados aqui (Figura 1).

Para cada um dos quatro sítios (Sítio $1=$ 1988; Sítio $2=2001$, Sítio $3=2007$, Sítio $4=$ 2010), foram estudados os campos graminóides e a vegetação sobre os afloramentos rochosos. Em cada um dos sítios destas duas fisionomias foram alocadas 50 parcelas de quatro $\mathrm{m}^{2}(2 \mathrm{~m}$ $\mathrm{x} 2 \mathrm{~m}$ ) para registro da composição florística $\mathrm{e}$ análise estrutural da vegetação. Assim, para cada um dos quatro sítios foram alocadas 100 parcelas, totalizando 400 parcelas para todo estudo $\left(1600 \mathrm{~m}^{2}\right.$ $=0,16$ ha). A distância entre as parcelas em um mesmo sítio foi sempre superior a $100 \mathrm{~m}$, sendo a distância maior entre as parcelas de diferentes sítios de $3,7 \mathrm{~km}$. A variação altitudinal foi menor do que $50 \mathrm{~m}$. As parcelas nos afloramentos rochosos foram dispostas de maneira à sempre incluir pelo menos uma ilha de vegetação, que poderia ser composta de uma única espécie. Neste caso foram consideradas ilhas de vegetação tanto nas bordas dos afloramentos quanto em seu interior.

\section{Análise de dados}

O levantamento florístico foi realizado em cada uma das parcelas, quando foram então registradas as espécies de angiospermas, samambaias e licófitas presentes. As espécies foram identificadas com auxílio de literatura (Aximoff \& Ribeiro 2012), e com apoio de especialistas do Instituto de Pesquisas Jardim Botânico do Rio de Janeiro e em consulta ao Herbário da mesma instituição. O nome das espécies e de seus autores, assim como a definição das espécies endêmicas estão de acordo com a lista de espécies da Flora do Brasil (http://floradobrasil.jbrj.gov.br/2012/). A categorização das espécies ameaçadas de extinção foi baseada na portaria do Ministério do Meio Ambiente $n^{\circ} 443$ (MMA 2014).

As variações fisionômicas foram observadas por meio da classificação das espécies em formas de vida e hábito. O hábito das espécies, isto é arbóreo, arbustivo, herbáceo, liana, epífitas e parasitas, foi definido pelo porte, lignificação 

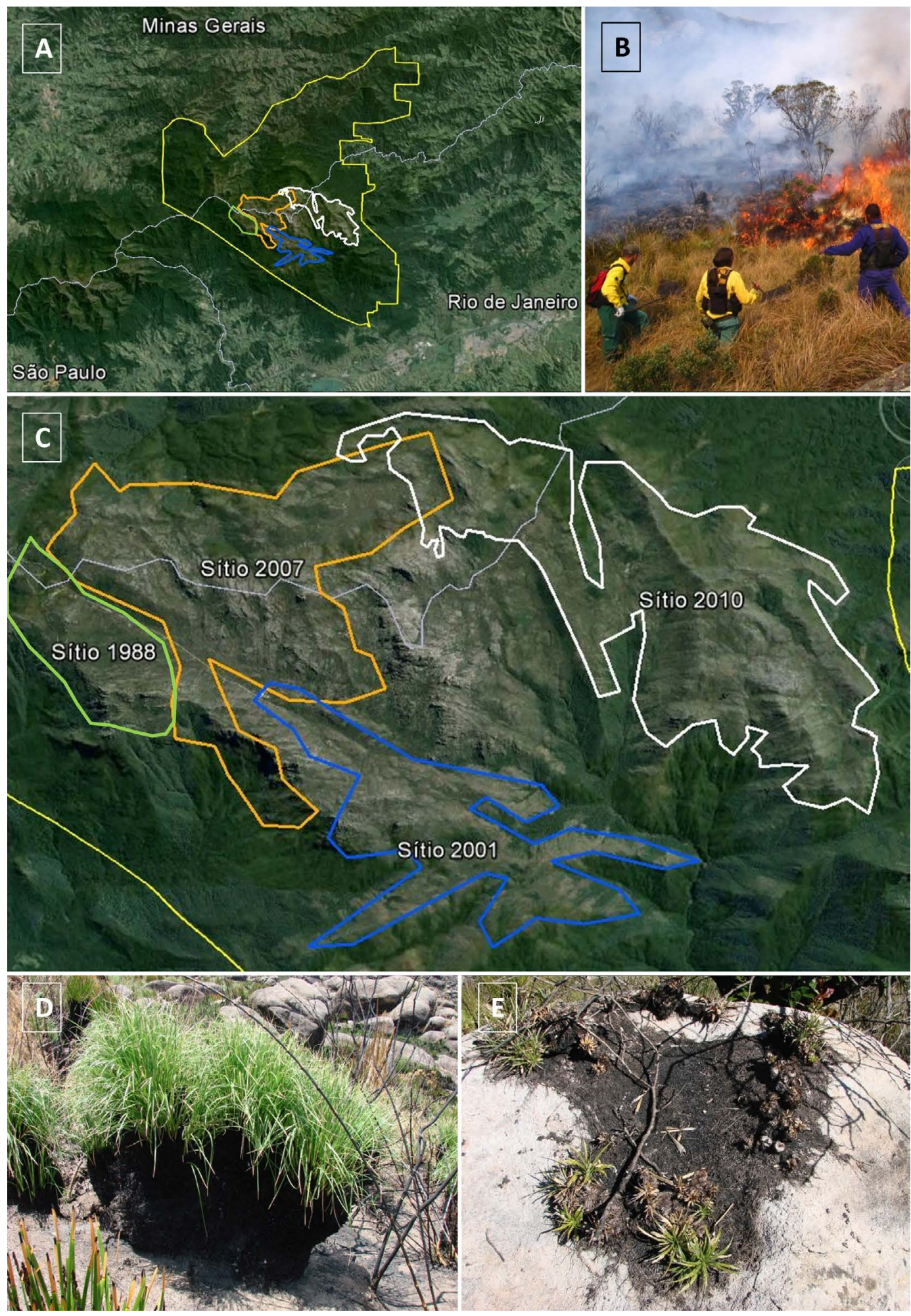

Figura 1. Limites do Parque Nacional do Itatiaia - PNI (linha amarela), dos Estados do Rio de Janeiro RJ, Minas Gerais - MG, São Paulo - SP (linha cinza), e das queimadas no interior do PNI (A), registro de combate a queimada em 2007 (B), localização dos sítios pesquisados (C), rebrotamento de Cortaderia modesta (Poaceae) pós-fogo (D), e ilha de vegetação queimada sobre afloramento rochoso (E).

Figure 1. Limits of the Itatiaia National Park - INP (yellow line), the states of Rio de Janeiro - RJ, Minas Gerais - MG, São Paulo - SP (gray line), and burning inside the INP (A), fighting fire in 2007 (B), location of the surveyed sites $(C)$, regrowth of Cortaderia modesta (Poaceae) post-fire $(D)$, and the island of burned vegetation on rocky outcrops (E). 
do caule e pelo sistema radicular. As formas de vida foram divididas em fanerófitas, caméfitas, hemicriptófitas, geófitas, terófitas e lianas, segundo adaptações de Raunkiaer (1934) e MuellerDombois \& Ellenberg (1974). De maneira à faciliar o entendimento, foram utilizadas três categorias de ambiente: campos graminóides, afloramentos rochosos e compartilhadas nos dois ambientes, para análise da riqueza e área de cobertura em relação a forma de vida.

As variações estruturais intra e entre sítios foram observadas por meio do levantamento dos parâmetros ecológicos: frequência e dominância (área de cobertura $\mathrm{em} \mathrm{m}^{2}$ ). A frequência foi medida pelo número de parcelas onde a espécie foi encontrada (NPe). A dominância foi calculada em quatro quadrículas de um $\mathrm{m}^{2}$ em cada parcela de quatro $\mathrm{m}^{2}$, com vistas a aumentar a precisão da estimativa visual da área de cobertura de cada espécie, ocupada pela projeção da parte aérea da planta (Brower \& Zar 1984). Assim, este parâmetro foi estimado a partir da área de cobertura das espécies $\left(\mathrm{m}^{2} / \mathrm{ha}\right)$, sendo que a soma dos valores de todas as áreas de cobertura das espécies pode ser superior a $1600 \mathrm{~m}^{2}$ devido a sobreposição das plantas. A área com superfície rochosa desnuda também foi medida. Para verificar a existência de diferenças entre os padrões florísticos e estruturais foram respectivamente utilizados o índice de similaridade de Sorensen e realizada uma análise de variância (ANOVA), seguido do teste $t$ de Tuckey para diferenças significativas $(\mathrm{p}<0,05)$, calculados pelo Biostat, versão 5.0 (Ayres et al. 2007, Zar 2010).

A densidade não foi determinada devido à dificuldade de individualização das plantas, principalmente daquelas formadoras de touceiras. Mueller-Dumbois \& Ellenberg (1974) consideraram as estimativas de cobertura e frequência suficientes para a análise descritiva de formações herbáceas ou baixo-arbustivas. Este procedimento foi adotado em estudos estruturais em outros campos de altitude (Caiafa \& Silva 2007) e em planícies costeiras arenosas (Menezes \& Araujo 2004).

\section{RESULTADOS}

Foram amostradas 183 espécies de plantas vasculares, distribuídas em 167 espécies de 56 famílias de angiospermas e 16 espécies de 7 famílias de samambaias e licófitas (Tabela 1). Dentre as 63 famílias registradas, nove destas são responsáveis por $40 \%$ da riqueza total amostrada, a saber: Asteraceae (31 espécies), Poaceae (13), Orchidaceae (9), Melastomataceae e Lamiaceae (8), Ericaceae e Pteridaceae (7), Rubiaceae (6) e Eriocaulaceae (5) (Tabela 1). A maior parte das famílias $(80,2 \%)$ é representada por até três espécies.

Foram amostradas 43 espécies exclusivas dos afloramentos rochosos. As famílias com maiores riquezas foram Asteraceae (15 espécies), Poaceae (11), Ericaceae (6), Orchidaceae (5) e Melastomataceae (4). Considerando apenas os campos graminóides, foram registradas 70 espécies, sendo as famílias com maior riqueza Asteraceae (27 espécies), Lamiaceae e Melastomataceae (8), Poaceae e Orchidaceae (7) e Ericaceae (6). O número de espécies compartilhadas entre afloramentos rochosos e campos graminóides foi 70 , quase $40 \%$ do total.

Foram registradas 57 espécies endêmicas da Serra da Mantiqueira (RJ, MG, SP) e pelo menos 15 espécies são restritas ao PNI: Baccharis itatiaiae, Chionolaena isabellae (Asteraceae), Begonia lanstyakii (Begoniaceae), Schlumbergera microsphaerica, S. opuntioides (Cactaceae), Valeriana glaziovii (Capriofilaceae), Leiothrix argyroderma (Eriocaulaceae), Geranium brasiliense (Geraniaceae), Pleroma cleistoflora (Melastomataceae), Fuchsia camposportoi (Onagraceae), Chusquea microphylla (Poaceae), Jamesonia cheilanthoides, J. brasiliensis (Pteridaceae), Buddleja speciosissima (Scrophulariaceae), Symplocos itatiaiae (Symplocaceae). Outras 12 espécies apresentam ocorrência no planalto do Itatiaia e em algum outro estado da região Sul.

Com base na lista atual de espécies da Flora Ameaçadas de Extinção no Brasil (Portaria MMA n $^{\circ} 443 / 2014$ ) foram registradas 20 espécies ameaçadas ( $11 \%$ do total), sendo 6 destas inclusas na categoria Vulnerável e outras 14 consideradas Em Perigo de Extinção (Tabela 1). Buddleja speciosissima (Scrophulariaceae) endêmica do planalto do Itatiaia, também foi indicada como ameaçada de extinção, segundo a penúltima lista (MMA 2008/ Anexo I). 
Tabela 1. Listagem das espécies registradas nos sítios queimados em diferentes períodos nos campos de altitude do Parque Nacional do Itatiaia. Abreviações: Fisiono - Fisionomias: AF - afloramento rochoso; CG - campo graminóide; Formas de Vida (FV): G - geófita; H - hemicriptófita; L - liana; F - fanerófita; C - caméfita; T - terófita. Háb - Hábito: Ab - arbustivo; Av - arbóreo; Hb -herbáceo; Ep - epífita; Ln - liana; $\mathrm{Pa}$ - parasita. End - Endemismo: $\mathrm{SE}=$ Sudeste, $\mathrm{PNI}=$ Parque Nacional do Itatiaia; Status de Ameaça (Portaria MMA n443/2014): EN - Em Perigo; VU - Vulnerável.

Table 1. List of species recorded in burned sites at different times in the high altitude grasslands in Itatiaia National Park. Abbreviations: Physiognomies: AF - rocky outcrop; $C G$ - graminoid field; life forms (FV): L storage organ; $H$ - hemicryptophytes; $L$ - liana; $F$ - phanerophytes; $C$ - camaephytes; $T$ - therophytes. Habit: Ab - shrubby; Av - arboreal; Hb - herb; Ep - epiphytic; Ln - liana; Pa - parasite. Endemism: SE = Southeast; Threat Status (MMA Ordinance \# 443/2014): EN - Endangered - in danger; VU - vulnerable.

\begin{tabular}{|c|c|c|c|c|c|c|c|c|c|}
\hline \multirow{2}{*}{ Família/Espécie } & \multirow[b]{2}{*}{ Fisiono } & \multirow[b]{2}{*}{ FV } & \multirow[b]{2}{*}{ Háb } & \multicolumn{4}{|c|}{ Sítios } & \multirow[b]{2}{*}{ End } & \multirow[b]{2}{*}{ Status } \\
\hline & & & & 2010 & 2007 & 2001 & 1988 & & \\
\hline \multicolumn{10}{|l|}{ Alstroemeriaceae } \\
\hline Alstroemeria foliosa Mart. & $\mathrm{AF} / \mathrm{CG}$ & G & $\mathrm{Hb}$ & $\mathrm{X}$ & $\mathrm{X}$ & $\mathrm{X}$ & $\mathrm{X}$ & & \\
\hline Alstroemeria isabelleana Herb. & $\mathrm{AF} / \mathrm{CG}$ & $\mathrm{G}$ & $\mathrm{Hb}$ & $\mathrm{X}$ & $\mathrm{X}$ & $\mathrm{X}$ & $\mathrm{X}$ & & \\
\hline \multicolumn{10}{|l|}{ Amaryllidaceae } \\
\hline Hippeastrum morelianum Lem. & $\mathrm{AF} / \mathrm{CG}$ & G & $\mathrm{Hb}$ & $\mathrm{X}$ & $\mathrm{X}$ & $\mathrm{X}$ & $\mathrm{X}$ & SE & VU \\
\hline \multicolumn{10}{|l|}{ Apiaceae } \\
\hline Eryngium glaziovianum Urb. & $\mathrm{AF} / \mathrm{CG}$ & $\mathrm{H}$ & $\mathrm{Hb}$ & $\mathrm{X}$ & $\mathrm{X}$ & $\mathrm{X}$ & $\mathrm{X}$ & SE & \\
\hline $\begin{array}{l}\text { Eryngium paniculatum Cav. \& Dombey ex } \\
\text { F.Delaroche }\end{array}$ & $\mathrm{AF} / \mathrm{CG}$ & $\mathrm{H}$ & $\mathrm{Hb}$ & $\mathrm{X}$ & $\mathrm{X}$ & $\mathrm{X}$ & $\mathrm{X}$ & & \\
\hline Eryngium sp. & $\mathrm{AF} / \mathrm{CG}$ & $\mathrm{H}$ & $\mathrm{Hb}$ & $\mathrm{X}$ & $\mathrm{X}$ & $\mathrm{X}$ & $\mathrm{X}$ & & \\
\hline \multicolumn{10}{|l|}{ Apocynaceae } \\
\hline Oxypetalum glaziovii (E. Fourn.) Fontella \& Marquete & CG & $\mathrm{L}$ & Ln & & & $\mathrm{X}$ & & SE & EN \\
\hline \multicolumn{10}{|l|}{ Aquifoliaceae } \\
\hline Ilex dumosa Reissek & $\mathrm{AF}$ & $\mathrm{F}$ & Av & & $\mathrm{X}$ & $\mathrm{X}$ & $\mathrm{X}$ & & \\
\hline \multicolumn{10}{|l|}{ Aspleniaceae } \\
\hline Asplenium serra Langsd. \& Fisch. & CG & $\mathrm{H}$ & $\mathrm{Hb}$ & & $\mathrm{X}$ & $\mathrm{X}$ & $\mathrm{X}$ & & \\
\hline Asplenium monanthes $\mathrm{L}$. & $\mathrm{CG}$ & $\mathrm{H}$ & $\mathrm{Hb}$ & & $\mathrm{X}$ & $\mathrm{X}$ & $\mathrm{X}$ & & \\
\hline \multicolumn{10}{|l|}{ Asteraceae } \\
\hline Achyrocline satureioides (Lam.) DC. & $\mathrm{AF} / \mathrm{CG}$ & $\mathrm{C}$ & $\mathrm{Hb}$ & $\mathrm{X}$ & $\mathrm{X}$ & $\mathrm{X}$ & $\mathrm{X}$ & & \\
\hline Baccharis altimontana G.Heiden et al. & $\mathrm{AF} / \mathrm{CG}$ & $\mathrm{F}$ & $\mathrm{Ab}$ & $\mathrm{X}$ & $\mathrm{X}$ & $\mathrm{X}$ & $\mathrm{X}$ & & \\
\hline Baccharis crispa Spreng. & CG & $\mathrm{F}$ & $\mathrm{Hb}$ & $\mathrm{X}$ & $\mathrm{X}$ & $\mathrm{X}$ & $\mathrm{X}$ & & \\
\hline Baccharis erigeroides DC. & $\mathrm{AF} / \mathrm{CG}$ & $\mathrm{F}$ & $\mathrm{Hb}$ & $\mathrm{X}$ & $\mathrm{X}$ & $\mathrm{X}$ & $\mathrm{X}$ & & \\
\hline Baccharis itatiaiae Wawra & $\mathrm{AF} / \mathrm{CG}$ & $\mathrm{F}$ & $\mathrm{Hb}$ & & $\mathrm{X}$ & & $\mathrm{X}$ & SE & \\
\hline Baccharis opuntioides Mart. ex Baker & CG & $\mathrm{F}$ & $\mathrm{Ab}$ & $\mathrm{X}$ & & $\mathrm{X}$ & & & \\
\hline Baccharis platypoda $\mathrm{DC}$ & $\mathrm{CG}$ & $\mathrm{F}$ & $\mathrm{Ab}$ & $\mathrm{X}$ & $\mathrm{X}$ & $\mathrm{X}$ & $\mathrm{X}$ & & \\
\hline Baccharis retusa DC. & $\mathrm{CG}$ & $\mathrm{F}$ & $\mathrm{Ab}$ & $\mathrm{X}$ & $\mathrm{X}$ & $\mathrm{X}$ & & & \\
\hline Baccharis stylosa Gardner & CG & $\mathrm{F}$ & $\mathrm{Ab}$ & $\mathrm{X}$ & $\mathrm{X}$ & $\mathrm{X}$ & $\mathrm{X}$ & & \\
\hline Baccharis uncinella DC. & $\mathrm{AF} / \mathrm{CG}$ & $\mathrm{F}$ & $\mathrm{Ab}$ & $\mathrm{X}$ & $\mathrm{X}$ & $\mathrm{X}$ & $\mathrm{X}$ & & \\
\hline Bidens segetum Mart.ex Colla & CG & $\mathrm{F}$ & $\mathrm{Hb}$ & & $\mathrm{X}$ & $\mathrm{X}$ & $\mathrm{X}$ & & \\
\hline Chionolaena capitata (Baker) S.E.Freire & $\mathrm{AF}$ & $\mathrm{F}$ & $\mathrm{Hb}$ & $\mathrm{X}$ & $\mathrm{X}$ & $\mathrm{X}$ & & & \\
\hline Chionolaena isabellae Baker & $\mathrm{AF} / \mathrm{CG}$ & $\mathrm{F}$ & $\mathrm{Hb}$ & & $\mathrm{X}$ & $\mathrm{X}$ & $\mathrm{X}$ & & \\
\hline Dendrophorbium sp. & $\mathrm{AF} / \mathrm{CG}$ & $\mathrm{F}$ & $\mathrm{Ab}$ & & $\mathrm{X}$ & $\mathrm{X}$ & $\mathrm{X}$ & & \\
\hline Leptostelma maxima D.Don & CG & $\mathrm{F}$ & $\mathrm{Hb}$ & & $\mathrm{X}$ & $\mathrm{X}$ & $\mathrm{X}$ & & \\
\hline Grazielia serrata (Spreng.) R.M.King \& H.Rob. & $\mathrm{AF}$ & $\mathrm{F}$ & $\mathrm{Hb}$ & $\mathrm{X}$ & & $\mathrm{X}$ & $\mathrm{X}$ & & \\
\hline
\end{tabular}




\begin{tabular}{|c|c|c|c|c|c|c|c|c|c|}
\hline \multirow{2}{*}{ Família/Espécie } & \multirow[b]{2}{*}{ Fisiono } & \multirow[b]{2}{*}{ FV } & \multirow[b]{2}{*}{ Háb } & \multicolumn{4}{|c|}{ Sítios } & \multirow[b]{2}{*}{ End } & \multirow[b]{2}{*}{ Status } \\
\hline & & & & 2010 & 2007 & 2001 & 1988 & & \\
\hline Gnaphalium sp. & CG & $\mathrm{H}$ & $\mathrm{Ab}$ & & $\mathrm{X}$ & $\mathrm{X}$ & $\mathrm{X}$ & & \\
\hline Graphistylis itatiaiae (Dusén) B.Nord. & CG & $\mathrm{F}$ & $\mathrm{Hb}$ & & $\mathrm{X}$ & $\mathrm{X}$ & $\mathrm{X}$ & & \\
\hline Grazielia intermedia (DC.) R.M.King \& H.Rob. & $\mathrm{AF} / \mathrm{CG}$ & $\mathrm{F}$ & $\mathrm{Hb}$ & & $\mathrm{X}$ & $\mathrm{X}$ & $\mathrm{X}$ & & \\
\hline $\begin{array}{l}\text { Hypochoeris brasiliensis (Less.) Benth. \& Hook.f } \\
\text { ex Griseb. }\end{array}$ & $\mathrm{CG}$ & $\mathrm{T}$ & $\mathrm{Hb}$ & & $\mathrm{X}$ & $\mathrm{X}$ & $\mathrm{X}$ & & \\
\hline Leptostelma maximum D.Don & $\mathrm{CG}$ & G & $\mathrm{Hb}$ & $\mathrm{X}$ & $\mathrm{X}$ & $\mathrm{X}$ & $\mathrm{X}$ & & \\
\hline Graphistylis argyrotricha (Dusén)B.Nord. & $\mathrm{AF}$ & $\mathrm{F}$ & $\mathrm{Hb}$ & $\mathrm{X}$ & $\mathrm{X}$ & $\mathrm{X}$ & $\mathrm{X}$ & & \\
\hline Senecio oleosus Vell. & $\mathrm{AF} / \mathrm{CG}$ & $\mathrm{F}$ & $\mathrm{Hb}$ & & & $\mathrm{X}$ & $\mathrm{X}$ & & \\
\hline Stevia camporum Baker & $\mathrm{AF} / \mathrm{CG}$ & G & $\mathrm{Hb}$ & & $\mathrm{X}$ & $\mathrm{X}$ & & SE & \\
\hline Symphyopappus compressus (Gardner) B.L.Rob. & $\mathrm{AF} / \mathrm{CG}$ & $\mathrm{F}$ & $\mathrm{Hb}$ & $\mathrm{X}$ & $\mathrm{X}$ & $\mathrm{X}$ & $\mathrm{X}$ & & \\
\hline Symphyopappus cuneatus Sch.Bip. ex Baker & CG & $\mathrm{F}$ & $\mathrm{Hb}$ & $\mathrm{X}$ & & $\mathrm{X}$ & & & \\
\hline $\begin{array}{l}\text { Symphyopappus itatiayensis (Hieron.) R.M.King \& } \\
\text { H.Rob. }\end{array}$ & $\mathrm{AF}$ & $\mathrm{F}$ & $\mathrm{Hb}$ & $\mathrm{X}$ & $\mathrm{X}$ & $\mathrm{X}$ & $\mathrm{X}$ & & \\
\hline Trixis glaziovii Baker & CG & $\mathrm{F}$ & $\mathrm{Hb}$ & & $\mathrm{X}$ & & & & VU \\
\hline Verbesina glabrata Hook. \& Arn. & $\mathrm{AF} / \mathrm{CG}$ & $\mathrm{F}$ & $\mathrm{Hb}$ & & $\mathrm{X}$ & $\mathrm{X}$ & $\mathrm{X}$ & & \\
\hline Wedelia subvelutina DC. & CG & $\mathrm{F}$ & $\mathrm{Hb}$ & $\mathrm{X}$ & $\mathrm{X}$ & $\mathrm{X}$ & & & \\
\hline \multicolumn{10}{|l|}{ Begoniaceae } \\
\hline Begonia lanstyakii Brade & $\mathrm{AF}$ & G & $\mathrm{Hb}$ & & & $\mathrm{X}$ & & PNI & \\
\hline \multicolumn{10}{|l|}{ Berberidaceae } \\
\hline Berberis glazioviana Brade & $\mathrm{CG}$ & $\mathrm{F}$ & $\mathrm{Ab}$ & & $\mathrm{X}$ & $\mathrm{X}$ & $\mathrm{X}$ & & \\
\hline Berberis laurina Thunb. & $\mathrm{CG}$ & $\mathrm{F}$ & $\mathrm{Ab}$ & $\mathrm{X}$ & & $\mathrm{X}$ & $\mathrm{X}$ & & \\
\hline \multicolumn{10}{|l|}{ Blechnaceae } \\
\hline Blechnum schomburgkii (Klotzsch) C.Chr. & $\mathrm{CG}$ & $\mathrm{H}$ & $\mathrm{Hb}$ & $\mathrm{X}$ & $\mathrm{X}$ & $\mathrm{X}$ & $\mathrm{X}$ & & \\
\hline \multicolumn{10}{|l|}{ Bromeliaceae } \\
\hline Fernseea itatiaiae (Wawra) Baker & $\mathrm{AF} / \mathrm{CG}$ & $\mathrm{H}$ & $\mathrm{Hb}$ & $\mathrm{X}$ & $\mathrm{X}$ & $\mathrm{X}$ & $\mathrm{X}$ & PNI & EN \\
\hline Nidularium marigoi Leme & $\mathrm{AF} / \mathrm{CG}$ & $\mathrm{H}$ & $\mathrm{Hb}$ & & $\mathrm{X}$ & $\mathrm{X}$ & & SE & \\
\hline Vriesea itatiaiae Wawra & $\mathrm{AF} / \mathrm{CG}$ & $\mathrm{H}$ & $\mathrm{Hb}$ & & $\mathrm{X}$ & & & SE & \\
\hline \multicolumn{10}{|l|}{ Cactaceae } \\
\hline Schlumbergera microsphaerica (K. Schum.) Hoevel & $\mathrm{AF}$ & $\mathrm{H}$ & $\mathrm{Hb}$ & & $\mathrm{X}$ & $\mathrm{X}$ & & & VU \\
\hline $\begin{array}{l}\text { Schlumbergera opuntioides (Loefgr. \& Dusén) } \\
\text { D.R.Hunt }\end{array}$ & $\mathrm{AF} / \mathrm{CG}$ & $\mathrm{H}$ & $\mathrm{Hb}$ & & $\mathrm{X}$ & & $\mathrm{X}$ & & VU \\
\hline \multicolumn{10}{|l|}{ Campanulaceae } \\
\hline Lobelia camporum Pohl & $\mathrm{CG}$ & $\mathrm{C}$ & $\mathrm{Hb}$ & $\mathrm{X}$ & $\mathrm{X}$ & $\mathrm{X}$ & $\mathrm{X}$ & & \\
\hline Siphocampylus longipedunculatus Pohl & $\mathrm{AF}$ & $\mathrm{C}$ & $\mathrm{Ab}$ & $\mathrm{X}$ & & $\mathrm{X}$ & $\mathrm{X}$ & & \\
\hline Siphocampylus westinianus (Thunb.) Pohl & $\mathrm{AF} / \mathrm{CG}$ & G & $\mathrm{Ab}$ & $\mathrm{X}$ & $\mathrm{X}$ & $\mathrm{X}$ & $\mathrm{X}$ & & \\
\hline \multicolumn{10}{|l|}{ Caprifoliaceae } \\
\hline Valeriana glaziovii Taub. & $\mathrm{CG}$ & $\mathrm{L}$ & Ln & & & $\mathrm{X}$ & $\mathrm{X}$ & PNI & \\
\hline \multicolumn{10}{|l|}{ Caryophyllaceae } \\
\hline Arenaria lanuginosa (Michx.) Rohrb. & $\mathrm{AF}$ & $\mathrm{C}$ & $\mathrm{Hb}$ & $\mathrm{X}$ & $\mathrm{X}$ & $\mathrm{X}$ & $\mathrm{X}$ & & \\
\hline Cerastium dicrotrichum Fenzl ex Rohrb. & $\mathrm{CG}$ & $\mathrm{C}$ & $\mathrm{Hb}$ & $\mathrm{X}$ & $\mathrm{X}$ & $\mathrm{X}$ & $\mathrm{X}$ & & \\
\hline Paronychia sp. & $\mathrm{AF}$ & $\mathrm{C}$ & $\mathrm{Hb}$ & & $\mathrm{X}$ & $\mathrm{X}$ & & & \\
\hline \multicolumn{10}{|l|}{ Clethraceae } \\
\hline Clethra scabra Pers. & $\mathrm{CG}$ & $\mathrm{F}$ & Av & $\mathrm{X}$ & $\mathrm{X}$ & $\mathrm{X}$ & $\mathrm{X}$ & & \\
\hline \multicolumn{10}{|l|}{ Commelinaceae } \\
\hline Tripogandra diuretica (Mart.) Handlos & $\mathrm{CG}$ & $\mathrm{F}$ & $\mathrm{Hb}$ & $\mathrm{X}$ & $\mathrm{X}$ & $\mathrm{X}$ & $\mathrm{X}$ & & \\
\hline
\end{tabular}




\begin{tabular}{|c|c|c|c|c|c|c|c|c|c|}
\hline \multirow{2}{*}{ Família/Espécie } & \multirow[b]{2}{*}{ Fisiono } & \multirow[b]{2}{*}{ FV } & \multirow[b]{2}{*}{ Háb } & \multicolumn{4}{|c|}{ Sítios } & \multirow[b]{2}{*}{ End } & \multirow[b]{2}{*}{ Status } \\
\hline & & & & 2010 & 2007 & 2001 & 1988 & & \\
\hline \multicolumn{10}{|l|}{ Cunnoniaceae } \\
\hline Weinmannia humilis Engl. & CG & $\mathrm{F}$ & Av & & $\mathrm{X}$ & $\mathrm{X}$ & $\mathrm{X}$ & & \\
\hline \multicolumn{10}{|l|}{ Cyperaceae } \\
\hline Carex brasiliensis A.St.-Hil. & $\mathrm{AF}$ & $\mathrm{H}$ & $\mathrm{Hb}$ & $\mathrm{X}$ & $\mathrm{X}$ & $\mathrm{X}$ & $\mathrm{X}$ & & \\
\hline Lagenocarpus triqueter (Boeckeler) Kuntze & $\mathrm{AF}$ & $\mathrm{H}$ & $\mathrm{Hb}$ & $\mathrm{X}$ & $\mathrm{X}$ & $\mathrm{X}$ & $\mathrm{X}$ & & \\
\hline Machaerina ensifolia (Boeckeler) T.Koyama & $\mathrm{AF} / \mathrm{CG}$ & $\mathrm{H}$ & $\mathrm{Hb}$ & $\mathrm{X}$ & $\mathrm{X}$ & $\mathrm{X}$ & $\mathrm{X}$ & & \\
\hline Rhynchospora sp. & $\mathrm{AF}$ & $\mathrm{H}$ & $\mathrm{Hb}$ & $\mathrm{X}$ & $\mathrm{X}$ & $\mathrm{X}$ & $\mathrm{X}$ & & \\
\hline \multicolumn{10}{|l|}{ Dioscoreaceae } \\
\hline Dioscorea demourae Uline ex R.Knuth & $\mathrm{AF}$ & $\mathrm{L}$ & Ln & & $\mathrm{X}$ & $\mathrm{X}$ & & & \\
\hline Dioscorea perdicum Taub. & CG & $\mathrm{L}$ & $\mathrm{Ln}$ & & $\mathrm{X}$ & $\mathrm{X}$ & $\mathrm{X}$ & PNI & \\
\hline \multicolumn{10}{|l|}{ Droseraceae } \\
\hline Drosera montana A.St.-Hil. & CG & $\mathrm{H}$ & $\mathrm{Hb}$ & $\mathrm{X}$ & $\mathrm{X}$ & $\mathrm{X}$ & $\mathrm{X}$ & & \\
\hline \multicolumn{10}{|l|}{ Dryopteridaceae } \\
\hline Elaphoglossum gayanum (Fée) T.Moore & $\mathrm{AF}$ & $\mathrm{H}$ & $\mathrm{Hb}$ & $\mathrm{X}$ & $\mathrm{X}$ & $\mathrm{X}$ & $\mathrm{X}$ & & \\
\hline \multicolumn{10}{|l|}{ Ericaceae } \\
\hline Agarista hispidula (DC.) Hook. ex Nied. & $\mathrm{AF} / \mathrm{CG}$ & $\mathrm{F}$ & $\mathrm{Ab}$ & & $\mathrm{X}$ & $\mathrm{X}$ & $\mathrm{X}$ & & \\
\hline Agarista oleifolia (Cham.) G.Don & $\mathrm{AF} / \mathrm{CG}$ & $\mathrm{F}$ & $\mathrm{Ab}$ & $\mathrm{X}$ & $\mathrm{X}$ & $\mathrm{X}$ & $\mathrm{X}$ & & \\
\hline Gaultheria eriophylla (Pers.) Sleumer ex Burtt & CG & $\mathrm{F}$ & $\mathrm{Ab}$ & $\mathrm{X}$ & $\mathrm{X}$ & $\mathrm{X}$ & $\mathrm{X}$ & SE & \\
\hline Gaultheria serrata (Vell.) Sleumer ex Kin.-Gouv. & $\mathrm{AF} / \mathrm{CG}$ & $\mathrm{F}$ & $\mathrm{Ab}$ & $\mathrm{X}$ & $\mathrm{X}$ & $\mathrm{X}$ & $\mathrm{X}$ & & \\
\hline Gaylussacia amoena Cham. & $\mathrm{AF} / \mathrm{CG}$ & $\mathrm{F}$ & $\mathrm{Ab}$ & & $\mathrm{X}$ & $\mathrm{X}$ & $\mathrm{X}$ & & \\
\hline Gaylussacia fasciculata Gardner & $\mathrm{AF} / \mathrm{CG}$ & $\mathrm{F}$ & $\mathrm{Ab}$ & & $\mathrm{X}$ & $\mathrm{X}$ & & SE & \\
\hline Gaylussacia jordanensis Sleumer & $\mathrm{AF} / \mathrm{CG}$ & $\mathrm{F}$ & $\mathrm{Ab}$ & $\mathrm{X}$ & $\mathrm{X}$ & $\mathrm{X}$ & $\mathrm{X}$ & SE & \\
\hline \multicolumn{10}{|l|}{ Eriocaulaceae } \\
\hline Actinocephalus polyanthus (Bong.)Sano & $\mathrm{AF} / \mathrm{CG}$ & $\mathrm{H}$ & $\mathrm{Hb}$ & $\mathrm{X}$ & $\mathrm{X}$ & $\mathrm{X}$ & $\mathrm{X}$ & & \\
\hline Eriocaulon majusculum Ruhland & $\mathrm{AF} / \mathrm{CG}$ & $\mathrm{H}$ & $\mathrm{Hb}$ & $\mathrm{X}$ & $\mathrm{X}$ & & $\mathrm{X}$ & SE & \\
\hline Leiothrix argyroderma Ruhland & $\mathrm{AF}$ & $\mathrm{H}$ & $\mathrm{Hb}$ & $\mathrm{X}$ & $\mathrm{X}$ & $\mathrm{X}$ & & PNI & \\
\hline Leiothrix beckii (Szyszl. ex Wawra) Ruhland & $\mathrm{AF} / \mathrm{CG}$ & $\mathrm{H}$ & $\mathrm{Hb}$ & $\mathrm{X}$ & & $\mathrm{X}$ & $\mathrm{X}$ & SE & \\
\hline Paepalanthus itatiaiensis Ruhland & CG & $\mathrm{H}$ & $\mathrm{Hb}$ & $\mathrm{X}$ & & $\mathrm{X}$ & $\mathrm{X}$ & PNI & \\
\hline \multicolumn{10}{|l|}{ Escalloniaceae } \\
\hline Escallonia laevis (Vell.) Sleumer & $\mathrm{AF} / \mathrm{CG}$ & $\mathrm{F}$ & $\mathrm{Ab}$ & $\mathrm{X}$ & $\mathrm{X}$ & $\mathrm{X}$ & $\mathrm{X}$ & & \\
\hline \multicolumn{10}{|l|}{ Euphorbiaceae } \\
\hline Croton dichrous Müll.Arg. & $\mathrm{AF} / \mathrm{CG}$ & $\mathrm{F}$ & $\mathrm{Av}$ & $\mathrm{X}$ & $\mathrm{X}$ & $\mathrm{X}$ & $\mathrm{X}$ & & \\
\hline Croton splendidus Mart. & $\mathrm{CG}$ & $\mathrm{F}$ & $\mathrm{Av}$ & & $\mathrm{X}$ & $\mathrm{X}$ & $\mathrm{X}$ & & \\
\hline \multicolumn{10}{|l|}{ Fabaceae } \\
\hline Lupinus arenarius Gardner & CG & $\mathrm{F}$ & $\mathrm{Ab}$ & & $\mathrm{X}$ & $\mathrm{X}$ & $\mathrm{X}$ & & \\
\hline Mimosa itatiaiensis Dusén & CG & $\mathrm{F}$ & $\mathrm{Ab}$ & & & & $\mathrm{X}$ & SE & \\
\hline Mimosa monticola Dusén & $\mathrm{AF} / \mathrm{CG}$ & $\mathrm{F}$ & $\mathrm{Ab}$ & & & & $\mathrm{X}$ & SE & \\
\hline Trifolium repens L. & CG & $\mathrm{H}$ & $\mathrm{Hb}$ & $\mathrm{X}$ & $\mathrm{X}$ & $\mathrm{X}$ & $\mathrm{X}$ & & \\
\hline \multicolumn{10}{|l|}{ Gentianaceae } \\
\hline Centaurium erythraea Rafn & CG & $\mathrm{H}$ & $\mathrm{Hb}$ & & $\mathrm{X}$ & $\mathrm{X}$ & $\mathrm{X}$ & & \\
\hline \multicolumn{10}{|l|}{ Geraniaceae } \\
\hline Geranium brasiliense Progel & CG & $\mathrm{H}$ & $\mathrm{Hb}$ & & & & $\mathrm{X}$ & PNI & \\
\hline \multicolumn{10}{|l|}{ Gesneriaceae } \\
\hline Sinningia gigantifolia Chautens & $\mathrm{AF}$ & $\mathrm{C}$ & $\mathrm{Hb}$ & & & $\mathrm{X}$ & $\mathrm{X}$ & SE & \\
\hline
\end{tabular}


.. Continuação

\begin{tabular}{|c|c|c|c|c|c|c|c|c|c|}
\hline \multirow{2}{*}{ Família/Espécie } & \multirow[b]{2}{*}{ Fisiono } & \multirow[b]{2}{*}{ FV } & \multirow[b]{2}{*}{ Háb } & \multicolumn{4}{|c|}{ Sítios } & \multirow[b]{2}{*}{ End } & \multirow[b]{2}{*}{ Status } \\
\hline & & & & 2010 & 2007 & 2001 & 1988 & & \\
\hline \multicolumn{10}{|l|}{ Gleicheniaceae } \\
\hline Dicranopteris nervosa (Kaulf.) Maxon & $\mathrm{AF}$ & $\mathrm{H}$ & $\mathrm{Hb}$ & $\mathrm{X}$ & $\mathrm{X}$ & $\mathrm{X}$ & $\mathrm{X}$ & & \\
\hline \multicolumn{10}{|l|}{ Griseliniaceae } \\
\hline Griselinia ruscifolia (Gay) Ball & $\mathrm{AF} / \mathrm{CG}$ & $\mathrm{F}$ & $\mathrm{Pa}$ & & $\mathrm{X}$ & $X$ & $X$ & & \\
\hline \multicolumn{10}{|l|}{ Iridaceae } \\
\hline Gelasine coerulea (Vell.) Ravenna & $\mathrm{AF} / \mathrm{CG}$ & $\mathrm{G}$ & $\mathrm{Hb}$ & $\mathrm{X}$ & $X$ & $\mathrm{X}$ & $\mathrm{X}$ & & \\
\hline Calydorea campestris (Klatt) Baker & $\mathrm{AF} / \mathrm{CG}$ & $\mathrm{H}$ & $\mathrm{Hb}$ & $X$ & $\mathrm{X}$ & $\mathrm{X}$ & $\mathrm{X}$ & & \\
\hline Sisyrinchium alatum Hook. & $\mathrm{AF} / \mathrm{CG}$ & $\mathrm{H}$ & $\mathrm{Hb}$ & $\mathrm{X}$ & $\mathrm{X}$ & $\mathrm{X}$ & $\mathrm{X}$ & & \\
\hline \multicolumn{10}{|l|}{ Juncaceae } \\
\hline Juncus microcephalus Kunth & $\mathrm{AF}$ & G & $\mathrm{Hb}$ & $\mathrm{X}$ & $\mathrm{X}$ & $\mathrm{X}$ & $\mathrm{X}$ & & \\
\hline \multicolumn{10}{|l|}{ Lamiaceae } \\
\hline Cunila galioides Benth. & $\mathrm{CG}$ & $\mathrm{F}$ & $\mathrm{Hb}$ & $\mathrm{X}$ & $\mathrm{X}$ & $\mathrm{X}$ & $\mathrm{X}$ & & \\
\hline $\begin{array}{l}\text { Hesperozygis myrtoides (A.St.-Hil. ex Benth.) } \\
\text { Epling }\end{array}$ & $\mathrm{AF} / \mathrm{CG}$ & $\mathrm{F}$ & $\mathrm{Hb}$ & $\mathrm{X}$ & & & $\mathrm{X}$ & $\mathrm{SE}$ & \\
\hline Lepechinia speciosa (A.St.-Hil. ex Benth.) Epling & $\mathrm{AF} / \mathrm{CG}$ & $\mathrm{F}$ & $\mathrm{Hb}$ & & $\mathrm{X}$ & $\mathrm{X}$ & & SE & \\
\hline Prunella vulgaris L. & CG & $\mathrm{F}$ & $\mathrm{Hb}$ & & $\mathrm{X}$ & $\mathrm{X}$ & $\mathrm{X}$ & & \\
\hline Rhabdocaulon coccineum (Benth.) Epling & $\mathrm{CG}$ & $\mathrm{F}$ & $\mathrm{Hb}$ & $X$ & & & $\mathrm{X}$ & SE & \\
\hline Salvia itatiaiensis Dusén & CG & $\mathrm{F}$ & $\mathrm{Ab}$ & & $\mathrm{X}$ & $\mathrm{X}$ & & SE & \\
\hline Salvia oligantha Dusén & $\mathrm{CG}$ & $\mathrm{F}$ & $\mathrm{Ab}$ & $\mathrm{X}$ & & & $\mathrm{X}$ & SE & \\
\hline Salvia sellowiana Benth. & $\mathrm{AF} / \mathrm{CG}$ & $\mathrm{F}$ & $\mathrm{Ab}$ & & $\mathrm{X}$ & & $\mathrm{X}$ & SE & \\
\hline \multicolumn{10}{|l|}{ Lentibulariaceae } \\
\hline Utricularia reniformis A.St.-Hil. & $\mathrm{AF}$ & $\mathrm{G}$ & $\mathrm{Hb}$ & $\mathrm{X}$ & $\mathrm{X}$ & $X$ & & & \\
\hline Utricularia tridentata Sylvén & $\mathrm{AF}$ & G & $\mathrm{Hb}$ & & $\mathrm{X}$ & $\mathrm{X}$ & & & VU \\
\hline \multicolumn{10}{|l|}{ Lythraceae } \\
\hline Cuphea sp. & $\mathrm{CG}$ & $\mathrm{F}$ & $\mathrm{Hb}$ & $\mathrm{X}$ & $\mathrm{X}$ & $\mathrm{X}$ & $\mathrm{X}$ & & \\
\hline \multicolumn{10}{|l|}{ Melastomataceae } \\
\hline Behuria parvifolia Cogn. & $\mathrm{CG}$ & $\mathrm{F}$ & $\mathrm{Ab}$ & & $\mathrm{X}$ & & & SE & \\
\hline Chaetostoma glaziovii Cogn. & $\mathrm{AF} / \mathrm{CG}$ & $\mathrm{C}$ & $\mathrm{Hb}$ & $\mathrm{X}$ & & $\mathrm{X}$ & $\mathrm{X}$ & $\mathrm{SE}$ & \\
\hline Huberia nettoana Brade & $\mathrm{CG}$ & $\mathrm{F}$ & $\mathrm{Ab}$ & & $\mathrm{X}$ & $\mathrm{X}$ & $\mathrm{X}$ & SE & \\
\hline Pleroma cleistoflora (Ule) P.J.F.Guim. et al. & $\mathrm{CG}$ & $\mathrm{H}$ & $\mathrm{Hb}$ & & $\mathrm{X}$ & $\mathrm{X}$ & & PNI & \\
\hline Leandra eichleri Cogn. & $\mathrm{AF} / \mathrm{CG}$ & $\mathrm{F}$ & $\mathrm{Ab}$ & $\mathrm{X}$ & $\mathrm{X}$ & $\mathrm{X}$ & $\mathrm{X}$ & & \\
\hline Leandra sulfurea (Naudin) Cogn. & $\mathrm{AF} / \mathrm{CG}$ & $\mathrm{F}$ & $\mathrm{Ab}$ & $\mathrm{X}$ & $\mathrm{X}$ & & $\mathrm{X}$ & & \\
\hline Tibouchina hospita Cogn. & $\mathrm{AF} / \mathrm{CG}$ & $\mathrm{F}$ & $\mathrm{Ab}$ & $\mathrm{X}$ & $\mathrm{X}$ & $\mathrm{X}$ & $\mathrm{X}$ & & \\
\hline Tibouchina sebastianopolitana Cogn. & $\mathrm{CG}$ & $\mathrm{F}$ & $\mathrm{Ab}$ & & $\mathrm{X}$ & $\mathrm{X}$ & & & \\
\hline \multicolumn{10}{|l|}{ Myrtaceae } \\
\hline Myrceugenia alpigena (DC.) Landrum & $\mathrm{AF} / \mathrm{CG}$ & $\mathrm{F}$ & Av & $\mathrm{X}$ & $\mathrm{X}$ & $\mathrm{X}$ & $\mathrm{X}$ & & \\
\hline \multicolumn{10}{|l|}{ Onagraceae } \\
\hline Fuchsia campos-portoi Pilg. \& G.K.Schulze & $\mathrm{CG}$ & $\mathrm{C}$ & $\mathrm{Ab}$ & & & $\mathrm{X}$ & $\mathrm{X}$ & SE & \\
\hline Fuchsia regia (Vell.) Munz & $\mathrm{AF} / \mathrm{CG}$ & $\mathrm{C}$ & $\mathrm{Hb}$ & $\mathrm{X}$ & $\mathrm{X}$ & $\mathrm{X}$ & $\mathrm{X}$ & & \\
\hline \multicolumn{10}{|l|}{ Orchidaceae } \\
\hline Coppensia blanchetii (Rchb.f.) Campacci & $\mathrm{AF} / \mathrm{CG}$ & G & $\mathrm{Hb}$ & & $\mathrm{X}$ & $\mathrm{X}$ & $\mathrm{X}$ & & \\
\hline Cranichis candida (Barb.Rodr.) Cogn. & $\mathrm{CG}$ & $\mathrm{G}$ & $\mathrm{Hb}$ & $\mathrm{X}$ & $\mathrm{X}$ & $\mathrm{X}$ & $\mathrm{X}$ & & \\
\hline
\end{tabular}




\begin{tabular}{|c|c|c|c|c|c|c|c|c|c|}
\hline \multirow{2}{*}{ Família/Espécie } & \multirow[b]{2}{*}{ Fisiono } & \multirow[b]{2}{*}{ FV } & \multirow[b]{2}{*}{ Háb } & \multicolumn{4}{|c|}{ Sítios } & \multirow[b]{2}{*}{ End } & \multirow[b]{2}{*}{ Status } \\
\hline & & & & 2010 & 2007 & 2001 & 1988 & & \\
\hline Cyclopogon apricus (Lindl.)Schltr. & CG & $\mathrm{G}$ & $\mathrm{Hb}$ & $\mathrm{X}$ & $\mathrm{X}$ & $\mathrm{X}$ & $\mathrm{X}$ & & \\
\hline Habenaria parviflora Lindl. & $\mathrm{AF} / \mathrm{CG}$ & G & $\mathrm{Hb}$ & $\mathrm{X}$ & $\mathrm{X}$ & $\mathrm{X}$ & $\mathrm{X}$ & & \\
\hline Habenaria rolfeana Schltr. & $\mathrm{AF} / \mathrm{CG}$ & G & $\mathrm{Hb}$ & $\mathrm{X}$ & $\mathrm{X}$ & $\mathrm{X}$ & $\mathrm{X}$ & & \\
\hline $\begin{array}{l}\text { Hadrolaelia mantiqueirae (Fowlie) Chiron \& } \\
\text { V.P.Castro }\end{array}$ & CG & G & Ep & $\mathrm{X}$ & $\mathrm{X}$ & $\mathrm{X}$ & $\mathrm{X}$ & & \\
\hline Pelexia itatiayae Schltr. & $\mathrm{CG}$ & G & $\mathrm{Hb}$ & $\mathrm{X}$ & $\mathrm{X}$ & $\mathrm{X}$ & $\mathrm{X}$ & & \\
\hline Prescottia montana Barb.Rodr. & $\mathrm{AF}$ & G & $\mathrm{Hb}$ & & $\mathrm{X}$ & $\mathrm{X}$ & $\mathrm{X}$ & & \\
\hline Zygopetalum maculatum (Kunth) Garay & $\mathrm{AF}$ & $\mathrm{G}$ & $\mathrm{Hb}$ & $\mathrm{X}$ & $\mathrm{X}$ & $\mathrm{X}$ & & & \\
\hline \multicolumn{10}{|l|}{ Orobanchaceae } \\
\hline Esterhazya eitenorum Barringer & $\mathrm{AF} / \mathrm{CG}$ & $\mathrm{F}$ & $\mathrm{Ab}$ & & & & $\mathrm{X}$ & SE & \\
\hline Esterhazya splendida J.C.Mikan & $\mathrm{AF} / \mathrm{CG}$ & $\mathrm{F}$ & $\mathrm{Ab}$ & & $\mathrm{X}$ & $\mathrm{X}$ & $\mathrm{X}$ & & \\
\hline \multicolumn{10}{|l|}{ Oxalidaceae } \\
\hline Oxalis confertissima A.St.-Hil. & $\mathrm{AF} / \mathrm{CG}$ & $\mathrm{H}$ & $\mathrm{Ab}$ & $\mathrm{X}$ & $\mathrm{X}$ & $\mathrm{X}$ & $\mathrm{X}$ & & \\
\hline Oxalis rupestris A.St.-Hil. & CG & $\mathrm{G}$ & $\mathrm{Hb}$ & $\mathrm{X}$ & $\mathrm{X}$ & $\mathrm{X}$ & $\mathrm{X}$ & & \\
\hline \multicolumn{10}{|l|}{ Piperaceae } \\
\hline Peperomia galioides Kunth & $\mathrm{AF}$ & $\mathrm{C}$ & $\mathrm{Hb}$ & $\mathrm{X}$ & $\mathrm{X}$ & $\mathrm{X}$ & & & \\
\hline \multicolumn{10}{|l|}{ Plantaginaceae } \\
\hline Plantago guilleminiana Decne. & CG & $\mathrm{H}$ & $\mathrm{Hb}$ & $\mathrm{X}$ & $\mathrm{X}$ & $\mathrm{X}$ & $\mathrm{X}$ & & \\
\hline \multicolumn{10}{|l|}{ Poaceae } \\
\hline Andropogon ternatus (Spreng.) Nees & $\mathrm{AF} / \mathrm{CG}$ & $\mathrm{H}$ & $\mathrm{Hb}$ & & $\mathrm{X}$ & $\mathrm{X}$ & $\mathrm{X}$ & & \\
\hline Andropogon lateralis Nees & CG & $\mathrm{H}$ & $\mathrm{Hb}$ & $\mathrm{X}$ & $\mathrm{X}$ & $\mathrm{X}$ & $\mathrm{X}$ & & \\
\hline Agrostis longiberbis Hack. ex L.B.Sm. & $\mathrm{AF}$ & $\mathrm{T}$ & $\mathrm{Hb}$ & $\mathrm{X}$ & & $\mathrm{X}$ & & & EN \\
\hline Agrostis lenis Roseng. et al. & $\mathrm{AF}$ & $\mathrm{H}$ & $\mathrm{Hb}$ & & $\mathrm{X}$ & & $\mathrm{X}$ & & EN \\
\hline Axonopus pellitus (Nees ex Trin.) Hitchc. \& Chase & $\mathrm{AF}$ & $\mathrm{H}$ & $\mathrm{Hb}$ & $\mathrm{X}$ & $\mathrm{X}$ & $\mathrm{X}$ & $\mathrm{X}$ & & \\
\hline Chascolytrum calotheca (Trin.) L.Essi et al. & $\mathrm{AF}$ & $\mathrm{H}$ & $\mathrm{Hb}$ & $\mathrm{X}$ & $\mathrm{X}$ & $\mathrm{X}$ & $\mathrm{X}$ & & \\
\hline Chascolytrum itatiaiae (Ekman) L.Essi et al. & $\mathrm{AF}$ & $\mathrm{H}$ & $\mathrm{Hb}$ & $\mathrm{X}$ & $\mathrm{X}$ & $\mathrm{X}$ & $\mathrm{X}$ & & EN \\
\hline Chusquea heterophylla Ness & $\mathrm{AF} / \mathrm{CG}$ & $\mathrm{F}$ & $\mathrm{Ab}$ & $\mathrm{X}$ & $\mathrm{X}$ & $\mathrm{X}$ & $\mathrm{X}$ & SE & EN \\
\hline Chusquea microphylla (Döll) L.G.Clark & $\mathrm{AF} / \mathrm{CG}$ & $\mathrm{F}$ & $\mathrm{Ab}$ & $\mathrm{X}$ & $\mathrm{X}$ & $\mathrm{X}$ & $\mathrm{X}$ & SE & \\
\hline Chusquea pinifolia (Nees) Nees & $\mathrm{AF} / \mathrm{CG}$ & $\mathrm{F}$ & $\mathrm{Ab}$ & $\mathrm{X}$ & $\mathrm{X}$ & $\mathrm{X}$ & $\mathrm{X}$ & SE & \\
\hline Cortaderia modesta (Döll) Hack & $\mathrm{AF} / \mathrm{CG}$ & $\mathrm{H}$ & $\mathrm{Hb}$ & $\mathrm{X}$ & $\mathrm{X}$ & $\mathrm{X}$ & $\mathrm{X}$ & & \\
\hline Danthonia secundiflora J.Presl & CG & $\mathrm{H}$ & $\mathrm{Hb}$ & $\mathrm{X}$ & $\mathrm{X}$ & $\mathrm{X}$ & $\mathrm{X}$ & & \\
\hline Paspalum polyphyllum Nees ex Trin. & $\mathrm{AF}$ & $\mathrm{H}$ & $\mathrm{Hb}$ & $\mathrm{X}$ & $\mathrm{X}$ & $\mathrm{X}$ & $\mathrm{X}$ & & \\
\hline \multicolumn{10}{|l|}{ Polygalaceae } \\
\hline Polygala brasiliensis $\mathrm{L}$. & $\mathrm{CG}$ & $\mathrm{C}$ & $\mathrm{Hb}$ & & $\mathrm{X}$ & $\mathrm{X}$ & $\mathrm{X}$ & & \\
\hline Polygala campestris Gardner & $\mathrm{CG}$ & $\mathrm{C}$ & $\mathrm{Hb}$ & $\mathrm{X}$ & $\mathrm{X}$ & $\mathrm{X}$ & $\mathrm{X}$ & & \\
\hline Polygala pulchella A.St.-Hil. \& Moq. & $\mathrm{AF}$ & $\mathrm{C}$ & $\mathrm{Hb}$ & & $\mathrm{X}$ & $\mathrm{X}$ & $\mathrm{X}$ & & \\
\hline \multicolumn{10}{|l|}{ Polygonaceae } \\
\hline Rumex acetosella $\mathrm{L}$. & $\mathrm{AF}$ & $\mathrm{T}$ & $\mathrm{Hb}$ & & $\mathrm{X}$ & $\mathrm{X}$ & $\mathrm{X}$ & & \\
\hline \multicolumn{10}{|l|}{ Polypodiaceae } \\
\hline Pleopeltis macrocarpa (Bory ex Willd.) Kaulf. & $\mathrm{AF}$ & $\mathrm{H}$ & $\mathrm{Hb}$ & & $\mathrm{X}$ & $\mathrm{X}$ & $\mathrm{X}$ & & \\
\hline $\begin{array}{l}\text { Serpocaulon catharinae (Langsd. \& Fisch.) } \\
\text { A.R.Sm. }\end{array}$ & $\mathrm{AF}$ & $\mathrm{H}$ & $\mathrm{Hb}$ & $\mathrm{X}$ & & $\mathrm{X}$ & $\mathrm{X}$ & & \\
\hline
\end{tabular}


... Continuação

\begin{tabular}{|c|c|c|c|c|c|c|c|c|c|}
\hline \multirow{2}{*}{ Família/Espécie } & \multirow[b]{2}{*}{ Fisiono } & \multirow[b]{2}{*}{ FV } & \multirow[b]{2}{*}{ Háb } & \multicolumn{4}{|c|}{ Sítios } & \multirow[b]{2}{*}{ End } & \multirow[b]{2}{*}{ Status } \\
\hline & & & & 2010 & 2007 & 2001 & 1988 & & \\
\hline Pleopeltis pleopeltidis (Fée) de la Sota & $\mathrm{AF} / \mathrm{CG}$ & $\mathrm{H}$ & $\mathrm{Hb}$ & $\mathrm{X}$ & & $\mathrm{X}$ & $\mathrm{X}$ & & \\
\hline \multicolumn{10}{|l|}{ Primulaceae } \\
\hline Myrsine gardneriana A.DC. & $\mathrm{AF} / \mathrm{CG}$ & $\mathrm{F}$ & $\mathrm{Av}$ & $\mathrm{X}$ & $\mathrm{X}$ & $\mathrm{X}$ & $\mathrm{X}$ & & \\
\hline \multicolumn{10}{|l|}{ Proteaceae } \\
\hline Roupala montana Aubl. & $\mathrm{AF} / \mathrm{CG}$ & $\mathrm{F}$ & Av & $\mathrm{X}$ & & $\mathrm{X}$ & $\mathrm{X}$ & & \\
\hline \multicolumn{10}{|l|}{ Pteridaceae } \\
\hline Campyloneurum angustifolium (Sw.) Fée & $\mathrm{CG}$ & $\mathrm{H}$ & $\mathrm{Hb}$ & $\mathrm{X}$ & & $\mathrm{X}$ & $\mathrm{X}$ & & \\
\hline Doryopteris feei Brade & $\mathrm{AF} / \mathrm{CG}$ & $\mathrm{H}$ & $\mathrm{Hb}$ & $\mathrm{X}$ & & $\mathrm{X}$ & $\mathrm{X}$ & & \\
\hline Doryopteris itatiaiensis (Fée) Christ & $\mathrm{AF}$ & $\mathrm{H}$ & $\mathrm{Hb}$ & & $\mathrm{X}$ & & $\mathrm{X}$ & SE & EN \\
\hline Doryopteris paradoxa (Fée) Christ & $\mathrm{AF}$ & $\mathrm{H}$ & $\mathrm{Hb}$ & & $\mathrm{X}$ & $\mathrm{X}$ & $\mathrm{X}$ & & VU \\
\hline Jamesonia cheilanthoides (Sw.) Christenh. & $\mathrm{CG}$ & $\mathrm{H}$ & $\mathrm{Hb}$ & & $\mathrm{X}$ & & & PNI & EN \\
\hline Jamesonia brasiliensis Christ & $\mathrm{CG}$ & $\mathrm{H}$ & $\mathrm{Hb}$ & & $\mathrm{X}$ & & & PNI & EN \\
\hline \multicolumn{10}{|l|}{ Rosaceae } \\
\hline Fragaria vesca $\mathrm{L}$. & $\mathrm{CG}$ & $\mathrm{H}$ & $\mathrm{Hb}$ & & $\mathrm{X}$ & $\mathrm{X}$ & $\mathrm{X}$ & & \\
\hline \multicolumn{10}{|l|}{ Rubiaceae } \\
\hline Coccocypselum condalia Pers. & $\mathrm{CG}$ & $\mathrm{C}$ & $\mathrm{Hb}$ & $\mathrm{X}$ & $\mathrm{X}$ & $\mathrm{X}$ & $\mathrm{X}$ & & \\
\hline Coccocypselum lymansmithii Standl. & $\mathrm{CG}$ & $\mathrm{C}$ & $\mathrm{Hb}$ & & $\mathrm{X}$ & $\mathrm{X}$ & $\mathrm{X}$ & & \\
\hline Galium humile Cham. \& Schltdl. & $\mathrm{CG}$ & $\mathrm{C}$ & $\mathrm{Hb}$ & $\mathrm{X}$ & $\mathrm{X}$ & $\mathrm{X}$ & $\mathrm{X}$ & & \\
\hline Hindsia glabra K.Schum. & $\mathrm{AF} / \mathrm{CG}$ & $\mathrm{C}$ & $\mathrm{Ab}$ & & $\mathrm{X}$ & & & PNI & EN \\
\hline Galium hypocarpium (L.) Endl. ex Griseb. & $\mathrm{AF}$ & $\mathrm{C}$ & $\mathrm{Hb}$ & $\mathrm{X}$ & $\mathrm{X}$ & $\mathrm{X}$ & $\mathrm{X}$ & & \\
\hline \multicolumn{10}{|l|}{ Scrophulariaceae } \\
\hline Buddleja speciosissima Taub. & $\mathrm{AF} / \mathrm{CG}$ & $\mathrm{F}$ & Av & & & & $\mathrm{X}$ & PNI & \\
\hline \multicolumn{10}{|l|}{ Selaginellaceae } \\
\hline Selaginella tenuissima Fée & $\mathrm{AF} / \mathrm{CG}$ & $\mathrm{C}$ & $\mathrm{Hb}$ & $\mathrm{X}$ & $\mathrm{X}$ & $\mathrm{X}$ & $\mathrm{X}$ & & \\
\hline \multicolumn{10}{|l|}{ Solanaceae } \\
\hline Solanum sp. & $\mathrm{AF}$ & G & $\mathrm{Ab}$ & $\mathrm{X}$ & $\mathrm{X}$ & $\mathrm{X}$ & $\mathrm{X}$ & & \\
\hline Solanum enantiophyllanthum Bitter & CG & $\mathrm{F}$ & $\mathrm{Ab}$ & & $\mathrm{X}$ & $\mathrm{X}$ & & SE & \\
\hline \multicolumn{10}{|l|}{ Symplocaceae } \\
\hline Symplocos itatiaiae Wawra & $\mathrm{AF}$ & $\mathrm{F}$ & $\mathrm{Ab}$ & & & & $\mathrm{X}$ & PNI & EN \\
\hline \multicolumn{10}{|l|}{ Theaceae } \\
\hline Laplacea fruticosa (Schrad.) Kobuski & $\mathrm{CG}$ & $\mathrm{F}$ & $\mathrm{Av}$ & & $\mathrm{X}$ & $\mathrm{X}$ & $\mathrm{X}$ & & \\
\hline \multicolumn{10}{|l|}{ Velloziaceae } \\
\hline Barbacenia gounelleana Beauverd & $\mathrm{AF}$ & $\mathrm{H}$ & $\mathrm{Hb}$ & $\mathrm{X}$ & $\mathrm{X}$ & $\mathrm{X}$ & $\mathrm{X}$ & SE & EN \\
\hline \multicolumn{10}{|l|}{ Verbenaceae } \\
\hline Verbena hirta Spreng. & $\mathrm{AF} / \mathrm{CG}$ & $\mathrm{F}$ & $\mathrm{Ab}$ & $\mathrm{X}$ & $\mathrm{X}$ & $\mathrm{X}$ & $\mathrm{X}$ & & \\
\hline \multicolumn{10}{|l|}{ Violaceae } \\
\hline Viola cerasifolia A.St.-Hil. & $\mathrm{AF}$ & $\mathrm{C}$ & $\mathrm{Hb}$ & $\mathrm{X}$ & $\mathrm{X}$ & $\mathrm{X}$ & $\mathrm{X}$ & & \\
\hline \multicolumn{10}{|l|}{ Winteraceae } \\
\hline Drimys brasiliensis Miers & $\mathrm{CG}$ & $\mathrm{F}$ & Av & $\mathrm{X}$ & $\mathrm{X}$ & $\mathrm{X}$ & $\mathrm{X}$ & & \\
\hline \multicolumn{10}{|l|}{ Xyridaceae } \\
\hline Xyris wawrae Heimerl & $\mathrm{AF}$ & $\mathrm{H}$ & $\mathrm{Hb}$ & & $\mathrm{X}$ & $\mathrm{X}$ & & SE & EN \\
\hline Xyris fusca L.A.Nilsson & CG & $\mathrm{H}$ & $\mathrm{Hb}$ & & $\mathrm{X}$ & $\mathrm{X}$ & & SE & EN \\
\hline
\end{tabular}


Em relação ao hábito das espécies amostradas, mais da metade são plantas herbáceas $(64,5 \%)$, seguidas pelas arbustivas $(25,7 \%)$, arbóreas $(6,6 \%)$, lianas $(2,2 \%)$, epífitas e parasitas (0,5\%). Analisando a porcentagem dos hábitos entre os sítios o teste Tukey apontou dominância das herbáceas em relação aos demais hábitos. $\mathrm{Na}$ análise de cada hábito, apenas no sítio 1988, a área de cobertura (dominância) das espécies arbóreas, com 18\% da área total, foi significativamente maior do que nos outros sítios que variaram de 8 a 11\% (Figura 2).

Foram identificadas diferenças significativas em relação às formas de vida nos campos graminóides e afloramentos rochosos, onde fanerófita e hemicriptófita apresentaram valores próximos a $50 \%$ das espécies. Nos campos graminóides, a forma de vida com maior número de espécies foi fanerófita $(47 \%)$, seguida por hemicriptófita $(27 \%)$, geófita e caméfita $(11 \%)$, liana $(4 \%)$ e terófita (1\%) (Figura 3). Nos afloramentos rochosos foi hemicriptófita $(42 \%)$, seguida por caméfita $(21 \%)$, geófita $(16 \%)$, fanerófita $(14 \%)$, terófita $(5 \%)$ e liana $(2 \%)$. Em relação às espécies compartilhadas nestes dois ambientes, houve predominância das fanerófitas com quase $60 \%$ das espécies. Em relação à dominância, houve diferença significativa com as espécies hemicriptófitas registradas em mais de $50 \%$ das amostras.

A riqueza identificada no sítio 2010 , equivalente a $57,4 \%$ das espécies registradas em toda área estudada, foi diferente daquela encontrada nos outros sítios com maior tempo de regeneração $(1988=83,1 \%, 2001=87,4 \%, 2007=$ $82,5 \%$ ). Entre quatro (sítio 2010) e sete anos (sítio 2007) pós-fogo, ocorreu um aumento de cerca de $30 \%$ na riqueza, que se manteve similar à área em regeneração por 25 anos (sítio 1988). Todas as espécies presentes no sítio 2010 ocorreram em um dos outros três sítios. Os valores do índice de similaridade florística de Sorensen intra sítio foram considerados elevados $(>0,70)$. Entre sítios, em geral, a similaridade entre afloramentos rochosos, e entre estes e campos graminóides, foi pouco abaixo dos valores apresentados pelos campos graminóides $(<0,70)$ (Tabela 2$)$.

As famílias mais frequentes e com maior cobertura (dominância) foram Poaceae, Cyperaceae e Asteraceae nos campos graminóides, e Asteraceae, Veloziaceae, Bromeliaceae e Poaceae nos afloramentos rochosos. As espécies Machaerina ensifolia (Cyperaceae) e Cortaderia

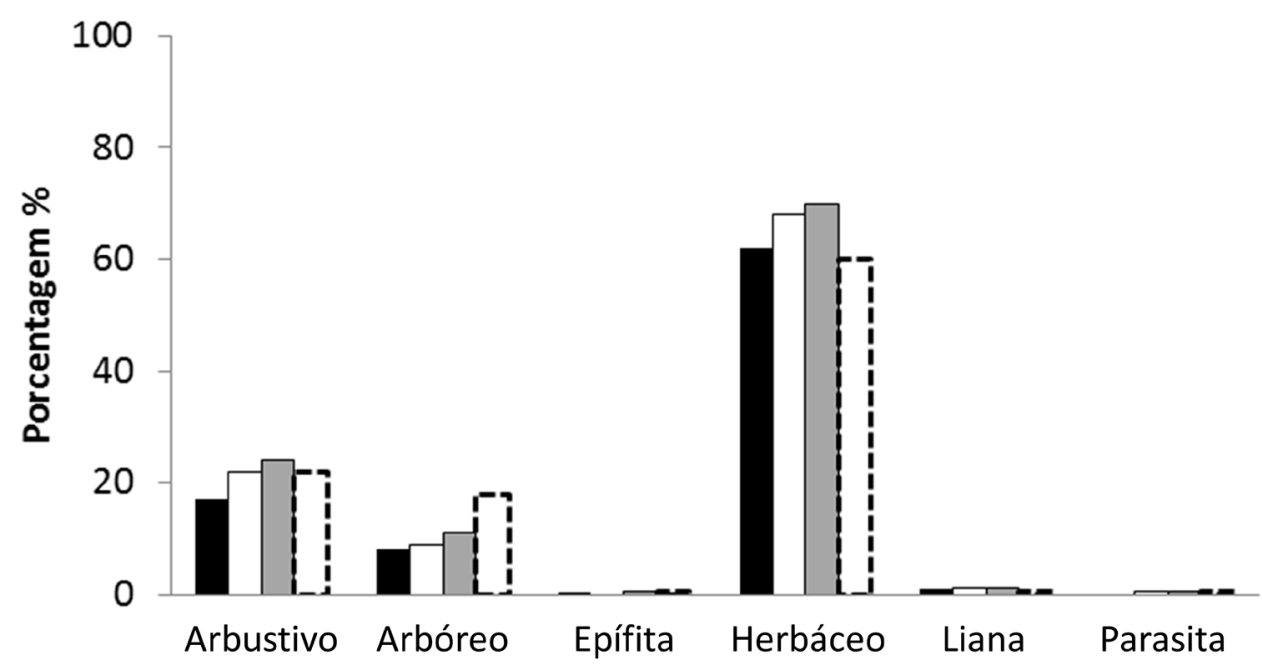

Hábito

Figura 2. Porcentagens da dominância (cobertura) por hábito das espécies amostradas nos sítios 2010 (barra preta), 2007 (barra branca), 2001 (barra cinza) e 1988 (barra tracejada) nos campos de altitude do Parque Nacional do Itatiaia.

Figure 2. Percentages of dominance (cover) the habit of the species sampled at sites 2010 (black bar), 2007 (white bar), 2001 (gray bar) and 1988 (hatched bar) in the high altitude grasslands in the Itatiaia National Park. 


\section{Riqueza}

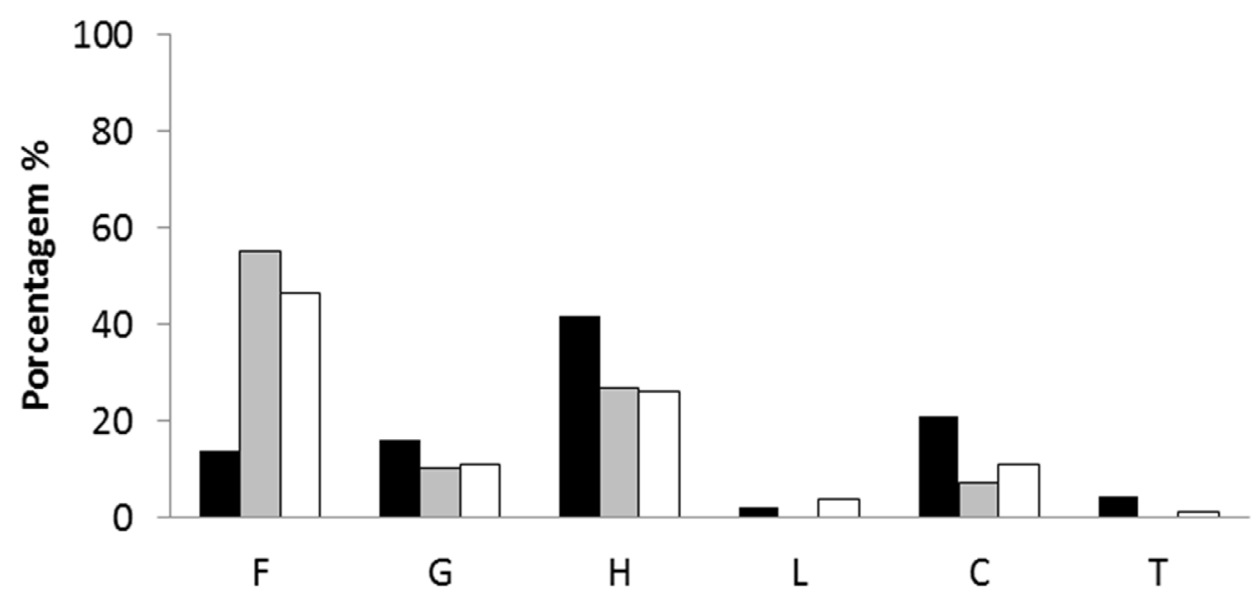

\section{Dominância $\left(\mathrm{m}^{2}\right)$}

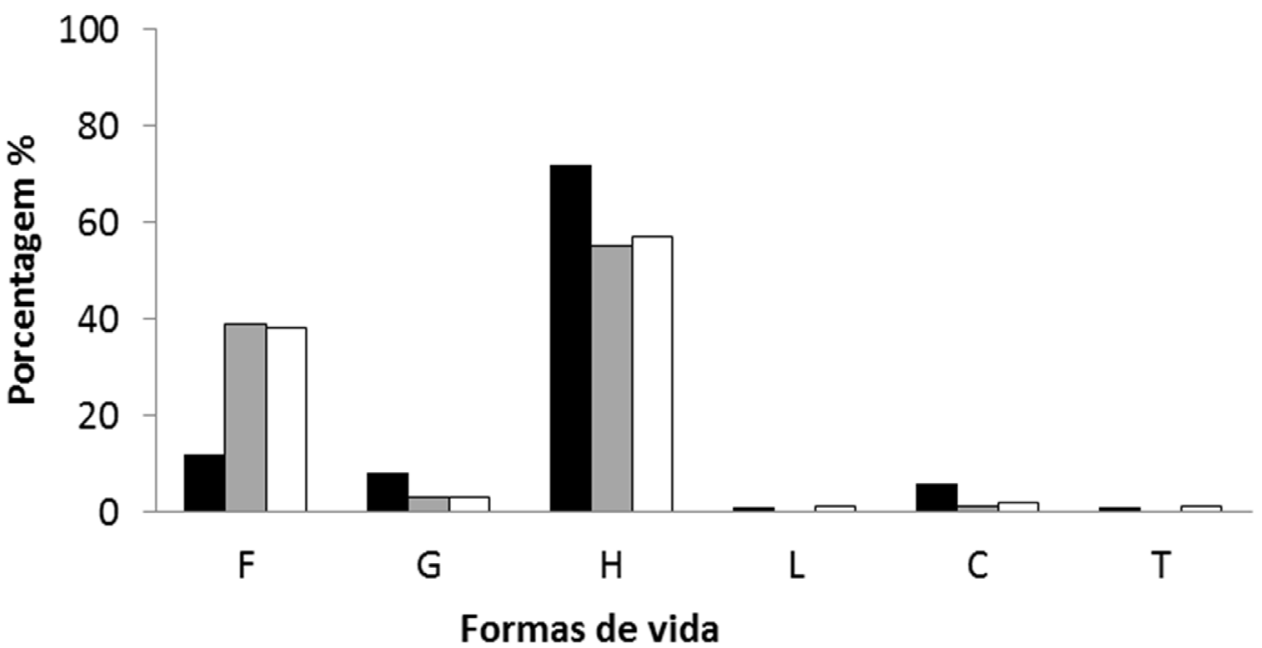

Figura 3. Porcentagens e dominância (cobertura) por formas de vida em afloramentos rochosos (barra preta), campos graminóides (barra branca) e nos dois ambientes (barra cinza). Abreviações das Formas de Vida: $\mathrm{G}$ geófita; $\mathrm{H}$ - hemicriptófita; L - liana; F - fanerófita; C - caméfita; T - terófita.

Figure 3. Percentages and dominance (cover) by forms of life on rocky outcrops (black bar), graminoid field (white bar) and the two environments (gray bar). Abbreviations of Life Forms: $G$ - storage organ; $H$ hemicryptophytes; L - liana; $F$ - phanerophytes; $C$ - camaephytes; $T$ - therophytes.

modesta (Poaceae) foram as mais frequentes, sendo registradas em mais de $90 \%$ das parcelas e com cerca de $80 \%$ da área de cobertura em todo o campo de altitude (Tabela 3). No sítio 2010, a área de cobertura ocupada pelas gramíneas dominantes é similar à registrada para o sítio 1988.

\section{DISCUSSÃO}

A riqueza registrada (183 espécies/0,16ha) equivale a cerca de $40 \%$ do total de espécies relatadas para todo o planalto do Itatiaia (i.e., $\mathrm{n}=415$, Martinelli 1996; $\mathrm{n}=550$, Safford 1999a), 
que apresenta cerca de $10 \mathrm{mil}$ ha de extensão, equivalente a cerca de $50 \%$ de toda a área de campos de altitude no Estado do Rio de Janeiro (Aximoff 2011). Este resultado vai de encontro com o encontrado por Ribeiro et al. (2007), que ao registrarem 114 espécies em 0,034 ha sobre afloramentos rochosos (sítio 2001), sugeriram que os valores da riqueza apontados em trabalhos anteriores estariam subestimados. Mesmo assim, apesar de não existirem listas de espécies em Martinelli (1996) e Safford (1999), os valores de riqueza considerados pelos autores para PNI são superiores aos registrados em outros campos de altitude no sudeste e sul do Brasil (Robim et al. 1990, Freitas \& Sazima 2006, Caiafa \& Silva 2007, Mocochinski \& Scheer 2008, Meireles et al. 2014). Considerando apenas os campos graminóides, a riqueza obtida foi semelhante à registrada para a mesma fisionomia no município de Camanducaia - MG (Meireles et al. 2014).

Embora o escasso número de levantamentos florísticos ainda não permita comparações precisas em relação à riqueza e composição de espécies entre outros campos de altitude do sudeste e sul do Brasil (Martinelli 2007), alguns padrões como famílias e gêneros dominantes já foram identificados. Considerando as famílias que representam quase $40 \%$ da riqueza total amostrada no PNI, dentre estas, Asteraceae e Poaceae estão entre as mais frequentes nos campos de altitude na Serra do Mar nos Estados do Rio de Janeiro (Safford 1999a), São Paulo (Freitas \& Sazima 2006), Paraná (Mocochinski \& Scheer 2008) e também na Serra da Mantiqueira nos Estados de São Paulo (Robim et al. 1990), Rio de Janeiro (Ribeiro et al. 2007) e Minas Gerais (Caiafa \& Silva 2007, Meireles et al. 2014).

A riqueza elevada dessas famílias se deve, em parte, à diversidade de hábitos e por ocuparem os afloramentos rochosos. Asteraceae e Poaceae foram as famílias mais ricas nos afloramentos rochosos do PNI, resultado similar ao registrado anteriormente por Ribeiro et al. (2007), e também para afloramentos rochosos em outros campos de altitude na Serra da Mantiqueira (Caiafa \& Silva 2007, Meireles et al. 2014). A ocorrência e dominância destas famílias nos campos de altitude podem também estar associadas a adaptações ao clima subtropical atual, que apresenta temperaturas abaixo de zero e geadas frequentes (Brade 1956; Segadas-Viana \& Dau 1965), como também a condições paleo-climáticas quaternárias (Azevedo 1962, Behling 1998).

A maior frequência das hemicriptófitas, com gemas e brotos de crescimento protegidos ao nível do solo, por escamas, folhas ou bainhas foliares, tem relação com a alta variação térmica diária e sazonalidade bem marcada, além do vento, frio,

Tabela 2. Índice de similaridade de Sorensen para as duas fisionomias estudadas nos sítios queimados em diferentes períodos nos campos de altitude do Parque Nacional do Itatiaia. Fisionomia: AF - Afloramento Rochoso; CG - Campos Graminóides. Letras diferentes sobreescritas representam diferenças significativas. Table 2. Sorensen similarity index for the two physiognomy studied in burned sites at different times in the high altitude grasslands in Itatiaia National Park. Physiognomy: AF - Outcrop Rocky; CG - Campos graminoid. Different letters represent significant differences overwritten.

\begin{tabular}{|c|c|c|c|c|c|c|c|c|c|c|}
\hline \multirow{2}{*}{\multicolumn{2}{|c|}{$\begin{array}{c}\text { Sítios / Fisionomia } \\
\text { (Riqueza) }\end{array}$}} & \multirow{3}{*}{$\frac{\boldsymbol{S}_{\boldsymbol{s}}}{0,78}$} & \multicolumn{2}{|c|}{2010} & \multicolumn{2}{|c|}{2007} & \multicolumn{2}{|c|}{2001} & \multicolumn{2}{|c|}{1988} \\
\hline & & & $\mathbf{A F}$ & $\mathbf{C A}$ & $\mathrm{AF}$ & $\mathrm{CA}$ & $\mathrm{AF}$ & $\mathrm{CA}$ & $\mathrm{AF}$ & $\mathrm{CA}$ \\
\hline \multirow{2}{*}{2010} & $\mathrm{AF}(71)^{\mathrm{a}}$ & & 1 & 0,58 & 0,59 & 0,57 & 0,60 & 0,56 & 0,63 & 0,66 \\
\hline & $\mathrm{CA}(79)^{\mathrm{a}}$ & 0,72 & & 1 & 0,57 & 0,75 & 0,65 & 0,75 & 0,66 & 0,75 \\
\hline \multirow{2}{*}{2007} & $\mathrm{AF}(95)^{\mathrm{b}}$ & 0,88 & & & 1 & 0,70 & 0,70 & 0,68 & 0,70 & 0,64 \\
\hline & $\mathrm{CA}(109)^{\mathrm{c}}$ & 0,73 & & & & 1 & 0,65 & 0,81 & 0,65 & 0,76 \\
\hline \multirow{2}{*}{2001} & $\mathrm{AF}(97)^{\mathrm{b}}$ & 0,81 & & & & & 1 & 0,73 & 0,75 & 0,68 \\
\hline & $\mathrm{CA}(120)^{\mathrm{c}}$ & 0,82 & & & & & & 1 & 0,66 & 0,84 \\
\hline \multirow{2}{*}{1988} & $\mathrm{AF}(94)^{\mathrm{b}}$ & 0,78 & & & & & & & 1 & 0,74 \\
\hline & $\mathrm{CA}(121)^{\mathrm{c}}$ & 0,79 & & & & & & & & 1 \\
\hline
\end{tabular}


Tabela 3. Parâmetros estruturais das espécies mais frequentes e dominantes $\left(\mathrm{m}^{2}\right)$ nos sítios queimados em diferentes períodos nos campos de altitude do Parque Nacional do Itatiaia. Abreviações: NPe - Número de parcelas onde foi encontrada a espécie; CT - Cobertura total da espécie $\left(\mathrm{m}^{2} / \mathrm{ha}\right)$.

Table 3. Structural parameters of the most frequent and dominant species $\left(\mathrm{m}^{2}\right)$ in the burned sites at different times in the highland plateaus in Itatiaia National Park. Abbreviations: NPE - number of plots where the species was found; $C T$ - Full coverage of the species $\left(\mathrm{m}^{2} / \mathrm{ha}\right)$.

\begin{tabular}{|c|c|c|c|c|c|c|c|}
\hline & \multirow{2}{*}{ Família } & \multirow{2}{*}{ Espécies } & \multirow{2}{*}{ Npe (\%) } & \multicolumn{4}{|c|}{ Cobertura total (m²/ha) } \\
\hline & & & & 2010 & 2007 & 2001 & 1988 \\
\hline \multirow{5}{*}{ 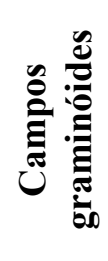 } & Cyperaceae & Machaerina ensifolia & $116(96,7)$ & 7.486 & 8.675 & 9.137 & 9.320 \\
\hline & Poaceae & Cortaderia modesta & $99(82,5)$ & 5.345 & 5.722 & 6.198 & 7.643 \\
\hline & Poaceae & Chusquea pinifolia & $98(81,7)$ & 3.532 & 3.841 & 4.233 & 4.421 \\
\hline & Asteraceae & Baccharis uncinella & $96(80,0)$ & 2.299 & 3.672 & 3.751 & 3.969 \\
\hline & Ericaceae & Gaylussacia amoema & $85(70,8)$ & 1.437 & 2.323 & 2.342 & 2.761 \\
\hline \multirow{5}{*}{ 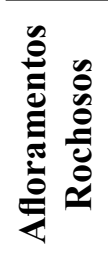 } & Bromeliaceae & Fernseea itatiaiae & $99(82,5)$ & 1.776 & 2.653 & 2.899 & 2.538 \\
\hline & Velloziaceae & Barbacenia gounelleana & $98(81,7)$ & 972 & 1.231 & 1.886 & 1.978 \\
\hline & Asteraceae & Baccharis stylosa & $82(68,3)$ & 786 & 876 & 921 & 834 \\
\hline & Poaceae & Axonopus pellitus & $79(65,8)$ & 544 & 632 & 722 & 765 \\
\hline & Alstroemeriaceae & Alstroemeria foliosa & $67(55,8)$ & 265 & 344 & 312 & 432 \\
\hline
\end{tabular}

geadas, dessecamento e mesmo fogo (Menezes \& Araujo 2004). Além das hemicriptófitas, asfanerófita e caméfitas, também foram registradas como formas de vidas mais frequentes nos campos de altitude das Serras do Brigadeiro (Caiafa \& Silva 2005) e dos Órgãos (Safford 2007).

Amaior frequência e dominância das espécies hemicriptófitas está diretamente relacionada com quatro espécies herbáceas, $M$. ensifolia (Cyperaceae) e C. modesta (Poaceae) nos campos graminóides, e $F$. itatiaiae (Bromeliaceae) e $B$. gounelleana (Velloziaceae) nos afloramentos rochosos. Cortaderia modesta também é uma das espécies dominantes nos campos de altitude do Parque Nacional da Serra dos Órgãos, juntamente com $C$. pinifolia, sendo as duas responsáveis por mais de $70 \%$ da área de cobertura entre espécies arbustivas e herbáceas na vegetação campestre (Safford 2001). A predominância de espécies herbáceas, seguida pelas espécies arbustivas, uma das características fisionômicas diferenciadas dos campos de altitude em relação à vegetação florestal do seu entorno, também está relacionado com as famílias mais ricas e frequentes, como Asteraceae, Cyperaceae, Ericaceae, dentre outras (Safford 1999, Mocochinski \& Scheer 2008, Meireles et al. 2014).
A capacidade de resiliência das comunidades vegetais nos campos graminóides e também nos afloramentos rochosos pode ser considerada eficiente, quando comparamos parâmetros como composição, riqueza e estrutura entre sítios com diferentes períodos de tempo pós-fogo. Por exemplo, o predomínio das espécies herbáceas e da forma de vida hemicriptófita foi similar para todos os sítios. Entre quatro e sete anos pós-fogo, mais de $80 \%$ das espécies registradas na composição florística estavam presentes nos sítios. Da mesma maneira, a riqueza foi similar nos sítios 2007, 2001 e 1988.

A dominância de C. modesta (Poaceae) e M. ensifolia (Cyperacea) foi similar em todos os sítios, incluindo o sítio 2010, comprovando que a regeneração destas duas espécies dominantes é rápida e persistente, mas não impede o aumento da riqueza da comunidade com o passar dos anos. O contínuo crescimento foliar do meristema intercalar destas espécies faz com que a posição sociológica seja reestabelecida rapidamente (Boldo et al. 2007).

Mesmo nos afloramentos rochosos, no sítio 2010, C. modesta e M. ensifolia ocorreram em maior abundância do que nos outros sítios. Ribeiro et al. (2007) também observaram sementes destas espécies em ilhas de vegetação não atingidas 
pelo fogo. Isto pode representar uma ameaça, aumentando a quantidade de biomassa combustível num ambiente naturalmente mais protegido do fogo. Contudo, 12 anos após a queimada no sítio 2001 , registrou-se $84,5 \%$ das espécies identificadas anteriormente em 1999-2000 por Ribeiro et al. (2007), mas ausência de C. modesta e M. ensifolia nos afloramentos rochosos.

O papel das espécies $F$. itatiaiae e $B$. gounelleana, dominantes e resistentes ao fogo, pode estar facilitando o processo de regeneração nas ilhas de vegetação (Medina et al. 2006, Aximoff 2011). Segundo Xavier et al. (2007), o sucesso de regeneração de comunidades vegetais é mais rápido quando as espécies dominantes na vegetação pré-fogo persistem pós-fogo. Além disso, Burke (2003) sugere que os afloramentos rochosos funcionem como fonte de propágulos para áreas campestres vizinhas.

Apesar do reestabelecimento da estrutura, composição e riqueza nos campos de altitude no PNI, algumas espécies endêmicas e ameaçadas de extinção não foram registradas após a passagem do fogo ou apresentaram baixa frequência comparando toda área estudada. No sítio 2001, por exemplo, a samambaia $D$. itatiaiensis, ameaçada de extinção e registrada por Ribeiro et al. (2007), não foi mais encontrada. Outras samambaias presentes em único sítio foram $J$. brasiliensis e $J$. cheilanthoides (Pteridaceae), ambas ameaçadas e com ocorrência restrita para Itatiaia. Brade (1956) também assinalou para a ausência de samambaias em áreas afetadas pelo fogo. Por outro lado, o sítio 2001 foi o único com ocorrência da endêmica Begonia lanstyakii (Begoniaceae). Outras espécies ameaçadas tiveram baixa frequência, como Oxypetalum glaziovii (Apocynaceae) e Xyris wawrae (Xyridaceae). Já Hindsia glabra (Rubiaceae) foi registrada apenas em uma parcela, justamente em uma área protegida do fogo, sobre afloramentos rochosos e entre blocos de pedra.

Dentre as nove espécies ameaçadas e endêmicas registradas em um único sítio, mais de $50 \%$ estão presentes no sítio 1988 , sendo esta a área com maior tempo de regeneração e menor ocorrência de queimadas. Segundo Barlow \& Peres (2008), quanto mais uma área florestal sofre queimadas, mais ela se diferencia da composição e estrutura original, o que parece ocorrer também para os campos de altitude. Apesar das espécies de campos de altitude serem em geral tolerantes ao fogo (Safford 2001), algumas menos resistentes ou de crescimento mais lento podem ser eliminadas.

Embora existam registros de paleoincêndios nos campos de altitude (Behling \& Safford 2010), as ocorrências atuais do fogo de origem natural são raras e quase sempre ocorrem nos meses de maior precipitação (Aximoff 2011). Considerando ainda o cenário atual de mudanças climáticas, onde há predições de eventos extremos com aumentos significativos de temperatura e extensão de períodos secos durante o inverno (Marengo et al. 2010, IPCC 2013), fatores diretamente relacionados com a ocorrência das maiores e/ou mais severas queimadas no PNI (Tomzinski et al. 2012), todos de origem antrópica, os riscos de novas ocorrências estarão cada vez maiores. Isto faz com que estudos sobre ecologia do fogo neste tipo de ambiente sejam prioritários.

Nesse sentido, o monitoramento da biomassa combustível, principalmente das espécies dominantes, que pode auxiliar na orientação do manejo visando a diminuição do estoque de combustível, tem sido utilizado como uma medida do grau de risco de incêndio no Cerrado (Silva et al. 2011). O fogo recorrente pode fazer com que estas espécies aumentem de densidade, tornando o ambiente cada vez mais favorável a novas ocorrências de fogo (Warman \& Moles 2009). Por outro lado, com o aquecimento global, a manutenção da fisionomia geral dos campos vai depender muito de fatores que agem contra o avanço das florestas. Assim, novas queimadas podem ser a chave para impedir a sucessão das formações florestais.

Contudo, considerando a riqueza de espécies endêmicas e ameaçadas de extinção que não apresentam resistência ao fogo conhecidas, existe necessidade de evitar a ocorrência do fogo. A inclusão de variáveis ambientais, de aspectos da estrutura funcional e trajetória sucessional da vegetação dos campos de altitude, além do mapeamento e de estudos reprodutivos e ecofisiológicos das espécies ameaçadas de extinção e da realização do fogo prescrito na borda destes ambientes e no entorno de capões de floresta, poderão ser fundamentais para melhor compreensão e detalhamento do processo de 
reestruturação da comunidade, auxiliando ainda no entendimento do comportamento do fogo na preservação da paisagem. Assim, é recomendável que se associe pesquisas científicas de longo prazo ao manejo do fogo nestas áreas.

Embora tenhamos registrado aqui um processo natural, rápido e eficiente de regeneração e, portanto, de manutenção dos campos de altitude pós-fogo, identificou-se também que algumas espécies endêmicas restritas e ameaçadas estão desaparecendo ou se tornando ainda mais raras. Acreditamos que os incêndios estejam contribuindo com isso. Arelação dos efeitos benéficos e maléficos do fogo nos campos de altitude ainda carecem de mais atenção, principalmente no sentido de garantir tanto a manutenção dos campos de altitude quanto a presença das espécies ameaçadas de extinção. Nesse sentido estudos populacionais, reprodutivos e ecofisiológicos dessas espécies tornam-se prioritários.

\section{AGRADECIMENTOS}

Ao Léo Nascimento, chefe de pesquisas do Parque Nacional do Itatiaia/ICMBio, pelo apoio logístico, aos revisores anônimos pelas importantes sugestões.

\section{REFERÊNCIAS}

Alves, R. J. V. 1994. Morphological age determination and longevity in some Vellozia populations in Brazil. Folia Geobotanica Phytotaxa Praha, 29, 55-59.

Aximoff, I. 2011. O que perdemos com a passagem do fogo pelos campos de altitude do Estado do Rio de Janeiro. Revista Biodiversidade Brasileira, Número Temático: Ecologia e Manejo do Fogo em Áreas Protegidas, 2, 180-200.

Aximoff, I., \& Ribeiro, K. T. (Orgs.) 2012. Guia de plantas: Planalto do Itatiaia. Rio de Janeiro: Editora Techinical Books, p. 224.

Aximoff, I., \& Rodrigues, R.C. 2011. Histórico dos incêndios florestais no Parque Nacional do Itatiaia. Ciência Florestal, 21 (1), 83-92.

Barlow, J., \& Peres, C. A. 2008. Fire-mediated dieback and compositional cascade in an Amazonian forest. Philosophical Transactions of the Royal Society B: Biological Sciences, 363, 1787-1794.

Brade, A.C. 1956. A flora do Parque Nacional do Itatiaia. Boletim do Parque Nacional do Itatiaia, 5, 1-114.

Behling, H. 1997. Late Quaternary vegetation, climate and fire history from the tropical mountain region of Morro de Itapeva, SE Brazil. Palaeogeography, Palaeoclimatology, Palaeoecology 129, 407-422.
Behling, H., Dupont, L., Safford, H. D., \& Wefer, G. 2007. Late-glacial and Holocene vegetation and climate dynamics in the Serra da Bocaina, southeastern Brazil. Quaternary International 131, 22-31.

Behling, H., \& Safford, H. D. 2010. Late-glacial and Holocene vegetation, climate and fire dynamics in the Serra dos Órgãos, Rio de Janeiro State, southeastern Brazil. Global Change Biology 16, 1661-1671.

Boldo, E. L., Simoni, G. L., Butzke, A., Lovatel, J. L., Scur, L,. \& Wasum, R. A. 2007. Avaliação da produtividade primária e da diversidade florística dos campos de cima da serra em diferentes alternativas de manejo de campo. Revista Brasileira Agroecologia 2, 1103-1106.

Brower, J. E., \& ZAR, J. H. 1984. Field and laboratory methods for general ecology. W.C. Brown Publishers, Boston.

Burke A. 2003. Inselbergs in a changing world - global trends. Divers Distrib 9, 375-383.

Caiafa, A. N., \& Silva, A. F. 2005. Composição florística e espectro biológico de um campo de altitude no Parque Estadual da Serra do Brigadeiro, Minas Gerais - Brasil. Rodriguesia, 56 (87), 163-173.

Caiafa, A. N. \& Silva, A. F. 2007. Structural analysis of the vegetation on a highland granitic rock outcrop in Southeast Brazil. Revista Brasileira de Botânica, 30 (4), 657-664.

CNCFlora 2011. Workshop para a elaboração da proposta de um Programa Nacional para a Pesquisa e Conservação em Ecossistemas de Montanhas. http://www.inot.org. br/artigo/Proposta_Plano\%20Nacional_Montanhas conabio_1.pd

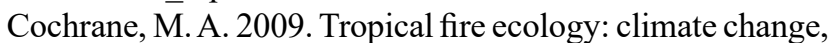
land use, and ecosystem dynamics. Chichester: Ed. Springer-Praxis. p. 628.

Conceição, A. A., \& Pirani, J. R. 2005. Delimitação de habitats em campos rupestres na Chapada Diamantina: substratos, composição florística e aspectos estruturais. Boletim de Botânica da Universidade de São Paulo, 23, 85-111.

Fidelis, A., Overbeck, G. E., Pillar, V. D., \& Pfadenhauer, J. 2008. Effects of disturbance on population biology of the rosette species Eryngium horridum Malme in grasslands in southern Brazil. Plant Ecology 195, 55-67.

França, H., Ramos Neto, M. B., \& Setzer, A. 2007. O fogo no Parque Nacional das Emas. Ministério do Meio Ambiente. Série Biodiversidade, p. 140.

Freitas, L., \& Sazima, M. 2006. Pollination biology in a tropical high-altitude grassland in Brazil: interactions at the community level. Annals Missouri Botanical Garde. 93, 465-516.

Hooper, E. R., Legendre, P., \& Condit, R. 2004. Factors affecting community composition of forest regeneration in deforested, abandoned land in Panamá. Ecology, 85, 3313-3326.

IBGE 2012. Manuel técnico da vegetação brasileira. Instituto Brasileiro de Geografia e Estatística, Rio de Janeiro. 271p. 
ICMBio 2013. Plano de Manejo do Parque Nacional do Itatiaia. http://www.Icmbio.gov.br/portal/images/stories/ docs-planos-de manejo/pm_parna_itatiaia_enc4.pdf.

Kolbek, J., \& Alves, R. J. V. 2008. Impacts of cattle, fire and wind in Rocky Savannas, Southeastern Brazil. Acta Universitatis Carolinae Environment, 22, 111-130.

Köppen, W. 1948. Climatologia tradicional. Traduzido para o Espanhol por Pedro Henchiehs Pérez, 1308-1315.

Marengo, J. A., Rusticucci, M., Penalba, O., \& Renom, M. 2010. An intercomparison of observed and simulated extreme rainfall and temperature events during the last half of the twentieth century: part 2: historical trends. Climatic Change 98, 509-529.

Martinelli, G. 1996. Campos de altitude. $2^{\text {a }}$ Ed., Editora Index. Rio de Janeiro. p. 160.

Martinelli, G. 2007. Mountain biodiversity in Brazil. Revista Brasileira de Botânica, 30, 587-597.

Medina, B. M. O., Ribeiro, K. T., \& Scarano, F. R. 2006. Plant-plant and plant-topography interactions on a rock outcrop at high altitude in southeastern Brazil. Biotropica $38,1-7$.

Meireles, L. D., Kinoshita, L. S., \& Shepherd, G. J. 2014. Floristic composition of high-montane vegetation in the district of Monte Verde (Camanducaia, Minas Gerais), Serra da Mantiqueira Meridional, Southeast Brasil. Rodriguésia, 65 (4), 831-859.

Meirelles, S. T., Pivello, V. R., \& Joly, C. A. 1999. The vegetation of granite rock outcrops in Rio de Janeiro, Brazil, and the need for its protection. Environmental Conservation 1, 10-20.

Menezes, L. F. T. D., \& Araujo, D. S. D. D. 2004. Regeneração e riqueza da formação arbustiva de Palmae em uma cronoseqüência pós-fogo na restinga da Marambaia, Rio de Janeiro, RJ, Brasil. Acta Botanica Brasilica. 18 (4), 771-780.

Miranda, H. S., Sato, M. N., Neto, W. N. \& Aires, F. S. 2009. Fires in the cerrado, the Brazilian savanna. In: Cochrane, M.A. Tropical Fire Ecology. pp. 427-450). Berlin: Springer Berlin Heidelberg.

MMA 2014. Ministério do Meio Ambiente: Portarias nº 443, 444, 445, de 17 de dezembro de 2014. Diário Oficial da União, Seção 1 (245), 110-130, 18 Dezembro 2014. 2014.

Mocochinski, A. Y., \& Scheer, M. B. 2008.Campos de Altitude na serra do mar paranaense: aspctos florísticos. Floresta. 4, 625-640.

Moreira, A. G. 2000. Effects of fire protection on savanna structure in central Brazil. Journal of Biogeography 27, 1021-1029.

Mueller-Dombois, D., \& Ellenberg, H. 1974. Aims and methods of vegetation ecology. New York, John Wiley \& Sons. p. 547.

Neves, S. P. S. \& Conceição, A. A. 2010. Campo rupestre recém-queimado na Chapada Diamantina, Bahia, Brasil: plantas de rebrota e sementes, com espécies endêmicas na rocha. Acta Botanica Brasilica, 24 (3), 697-707.

Ribeiro, K. T., Medina, B. M. O., \& Scarano, F. R. 2007. Species Composition and Biogeographic Relations of the
Rock Outcrop Flora on the High Plateau of Itatiaia, SEBrazil, Revista Brasileira de Botânica, 30 (4), 623-639.

Robim, M. J., Pastore, J. A., Aguiar, O. T., \& Baitello, J. B. 1990. Flora arbóreo arbustiva e herbácea do Parque Estadual de Campos do Jordão, SP. Revista do Instituto Florestal 2: 31-53.

Safford, H. D. 1999a. Brazilian páramos I. Introduction to the physical environment and vegetation of the campos de altitude. The Journal of Biogeography 26, 713-738.

Safford, H. D. 1999b. Brazilian páramos II. Macro- and mesoclimate of the campos de altitude and affinities with high mountain climates of the tropical Andes and Costa Rica. The Journal of Biogeography 26, 739-760.

Safford, H. D. 1999c. Notas sobre a ecologia e preservação de Nothochilus coccineus (Scrophulariaceae), espécie endêmica ao Maciço do Caparaó, ES/MG. [Notes on the ecology and conservation of Nothochilus coccineus (Scrophulariaceae), species endemic to the Caparaó Massif, ES/MG, Brazil]. Acta Botânica Brasilica 13 (2), 175-185.

Safford, H. D. 2001. Brazilian páramos III. Patterns and rates of postfire regeneration in the campos de altitude. Biotropica 33, 282-302.

Safford, H. D. 2007. Brazilian páramos IV. Phytogeography of the campos de altitude. Journal of Biogeography 34, $1701-1722$.

Safford, H. D., \& Martinelli, G. 2000. Southeast Brazil. In: Inselbergs: Biotic diversity of isolated rock outcrops in tropical and temperate regions (ed. by W. Barthlott \& S. Porembski), pp. 339-389. Ecological Studies No. 146. Springer-Verlag, Berlin.

Scarano, F. R. 2002. Structure, Function and Floristic Relationships of Plant Communities in Stressful Habitats Marginal to the Brazilian Atlantic Rainforest, Annals of Botany, 90 (4), 517-524.

Scarano, F. R., Duarte, H. M., Ribeiro, K. T., Rodrigues, P. J. F., \& Barcellos, E. M. B. 2001. Four sites with contrasting environmental stress in southeastern Brazil: relations of species, life form diversity, and geographic distribution to ecophysiological parameters. Botanical Journal of the Linnean Society, 136(4), 345-364.

Silva, D. M., Loiola, P. P., Rosatti, N. B., Silva, I. A., Cianciaruso, M. V., \& Batalha, M. A. 2011. Os efeitos dos regimes de fogo sobre a vegetação de Cerrado no Parque Nacional das Emas, GO: considerações para a conservação da diversidade. Biodiversidade Brasileira, (2), 26-39.

Silva, I. A., Carvalho, G. H., Loiola, P. P., Cianciaruso, M. V., \& Batalha, M.A. 2010. Herbaceous and shrubby species co-occurrences in Brazilian savannas: the roles of fire and chance. Community Ecology, 1, 97-104.

Stocker, T. F., Qin, D., Plattner, G. K., Tignor, M., Allen, S. K., Boschung, J., Nauels, A., Xia, Yu., Bex, V.\& Midgley, P. M. 2013. IPCC, 2013: climate change 2013: the physical science basis. Contribution of working group I to the fifth assessment report of the intergovernmental panel on climate change. Cambridge: Cambridge University Press. p. 27 
Tomzhinski, G. W., Ribeiro, K. T., \& Fernandes, M. C. 2012. Análise Geoecológica dos Incêndios Florestais no Parque Nacional do Itatiaia. Boletim de Pesquisa do Parque Nacional do Itatiaia. 65p.

Veríssimo, P., N., H. D. Safford, \& H. Behling. 2012. Holocene vegetation and fire history of the Serra do Caparaó, SE Brazil. The Holocene 22, 1243-1250.
Vieira, D. L. \& Scariot, A. 2006. Principles of natural regeneration of tropical dry forests for restoration. Restoration Ecology, 14(1), 11-20.

Warman, L., \& Moles, A.T. 2009. Alternative stable states in Australia's wet tropics: a theoretical framework for the field data and a field-case for the theory. Landscape Ecology, 24, 1-13.

Submetido em: $12 / 03 / 2015$ Aceito em: $23 / 03 / 2016$ 